\title{
Effective toughness of disordered brittle solids: a homogenization framework
}

\author{
Mathias Lebihain ${ }^{\mathrm{a}, \mathrm{b}}$, Laurent Ponson ${ }^{\mathrm{a}}$, Djimédo Kondo ${ }^{\mathrm{a}}$, Jean-Baptiste Leblond ${ }^{\mathrm{a}}$ \\ ${ }^{a}$ Institut Jean le Rond D'Alembert (Sorbonne Université, UPMC, CNRS UMR 7190), 4 Place Jussieu, 75005 Paris, \\ France \\ ${ }^{b}$ Laboratoire Navier (Université Paris-Est, ENPC, IFSTTAR, CNRS UMR 8205), 6-8 avenue Blaise Pascal, 77455 \\ Marne-la-Vallée, France
}

\begin{abstract}
This paper addresses the question of the homogenization of fracture properties for three-dimensional disordered brittle solids. The effective toughness, identified as the minimum elastic energy release rate required to ensure crack growth, is predicted from a semi-analytical framework inspired by both micromechanics and statistical physics that encompasses the decisive influences of both the material disorder and the mechanisms of interaction between a crack and heterogeneities. Theoretical predictions are compared to numerical values of the effective toughness that are computed with the fracture mechanics based semi-analytical method of Lebihain et al. (2020). Based on a perturbative approach of Linear Elastic Fracture Mechanics, this method allows for the efficient computation of crack propagation under tensile Mode I loading in composite material containing several millions of inclusions, where the crack interacts with them through two mechanisms : crossing, wherein the crack penetrates the inclusion, and by-pass, wherein the crack wanders out-of-plane and follows the inclusion/matrix interface. We show that our homogenization procedure provides an accurate prediction of the homogenized fracture properties for a broad range of microstructural parameters such as the inclusions toughness, their density or their shape. This original theoretical framework constitutes a powerful mean to bridge the microstructural parameters of materials with their crack growth resistance, beyond the particular cases considered in the performed simulations. As a result, it provides new strategies for the rational design of optimized brittle composites with tailored fracture properties.
\end{abstract}

Keywords: Brittle fracture, homogenization theory, disordered materials, effective toughness, large-scale simulations, rational design

\section{Introduction}

Crack propagation is the dominant mode of failure of materials under traction. However, our understanding of the impact of microstructural parameters on the resistance to crack growth is still largely incomplete. Recently, the boom of additive manufacturing techniques, and the emergence of bio-source and recycled composite materials driven by urging environmental concerns, have increased further the need for rationalizing the failure properties of micro-structured solids

${ }^{*}$ Corresponding author : mathias.lebihain@enpc.fr Preprint submitted to Journal of the Mechanics and Physics of Solids 
(Reis, 2006; Dimas et al., 2013; Mirkhalaf et al., 2014; Chandler et al., 2016; Wang and Xia, 2017).

Beyond fracture problems, predicting the macroscopic properties of materials from the knowledge of its constituents at a microscopic or mesoscopic scale has always been the Holy Grail pursued by materials science (Torquato, 2002), for it provides building bricks for the understanding of complex material behaviors as well as for the development of tailor-made optimized materials. A powerful theoretical framework has been developed within the mechanics community to estimate the overall response of composite materials from their microstructure, for elastic media (Hashin and Shtrikman, 1963; Hill, 1963; Herve and Zaoui, 1993; Ponte-Castañeda and Willis, 1995; Milton, 2002) as well as cracked ones (Ponte-Castañeda and Willis, 1995; Pensée V. et al., 2002; Deudé V. et al., 2002; Dormieux et al., 2006). Yet, such approaches fail to predict the toughness of materials that emerges from the propagation of a crack. Brittle fracture is indeed a very peculiar problem that exhibits unique features : it is (i) a dissipative evolution problem that (ii) localizes at the crack tip and (iii) is coupled with a structural one so that the definition of intrinsic effective fracture properties independent of the embedding structure is challenged. A comprehensive theoretical framework for the homogenization of brittle fracture properties should thus provide (i) tools to quantitatively model crack propagation in disordered materials as well as (ii) an accurate description of the dissipative processes involved at the crack tip during the interaction of a crack with material heterogeneities. One should finally estimate homogenized fracture properties from (iii) the resolution of the structural problem. The stress singularity at the crack tip enhances at the large scale the local mechanisms of interaction between a crack and inclusions, which have been extensively studied in the past few decades (e.g. crack trapping (Gao and Rice, 1989; Bower and Ortiz, 1990; Vasoya et al., 2016), crack deflection (Faber and Evans, 1983; He and Hutchinson, 1989; Clayton and Knap, 2014; Brach et al., 2019; Lebihain et al., 2020), crack shielding by micro-cracking (Evans and Faber, 1981; Ortiz, 1987), crack denucleation/renucleation (Leguillon et al., 2006; Hossain et al., 2014; Wang and Xia, 2017) and crack bridging (Bower and Ortiz, 1991; Mirkhalaf et al., 2014)). These pioneering works provide a detailed description of the conditions under which one mechanism prevails over one another as well as its ultimate contribution to the material reinforcement. Yet, they are generally restricted to a two-dimensional or periodic setting, neglecting thus the decisive impact of material disorder. As the proposed study will show, disordered arrangements of inlusions give rise to collective phenomena that cannot be captured by a single inclusion problem (see also (Alava et al., 2006), (Bonamy and Bouchaud, 2011) and (Ponson, 2016)). Roux et al. (2003), Roux and Hild (2008) and Patinet et al. (2013b) proposed a semi-analytical self-consistent approach that takes into account the influence of material disorder on the effective toughness in a three-dimensional setting. Démery et al. (2014a) and Démery et al. (2014b) addressed the same problem with tools borrowed from statistical physics (Larkin and Ovchinnikov, 1979) to develop a theoretical framework, which allows for analytical predictions of the homogenized fracture properties from the resolution of the structural problem. If these works constitute major advances in the field of homogenization of brittle fracture properties, they are nonetheless restricted to coplanar crack propagation and can only account for the crossing mechanism of interaction between a crack and tough inclusions.

In the present study, we propose a comprehensive theoretical homogenization framework that allows for the definition of intrinsic effective fracture properties. Based on concepts borrowed from statistical physics, this framework fully encompasses the influence of the interaction 
mechanisms localized at the crack tip and the collective effects controlling crack propagation in three-dimensional disordered materials. Theoretical predictions of the proposed model are successfully compared to numerical results extracted from simulations of crack propagation in large-scale disordered materials containing millions of tough inclusions, where the crack interacts either through a crossing or a by-pass mechanism (Lebihain et al., 2020). The proposed approach allows for an accurate prediction of the homogenized fracture properties for a broad range of microstructural parameters such as the inclusion toughness, its density, and its shape. Beyond the particular cases considered in the performed simulations, we explain why this original theoretical framework constitutes a powerful and versatile mean to bridge the microstructural parameters of materials with their crack growth resistance.

The paper is organized as follows : in Section 2, we briefly recall the main ingredients behind the perturbative approach proposed by Lebihain et al. (2020) that allows for large-scale simulations of crack propagation in brittle composites with spatial toughness heterogeneities. Section 3 is devoted to the effective toughness of heterogeneous materials. We first challenge the competing definitions of the effective fracture properties and highlight a scale-separation condition under which they all converge to an intrinsic value, defined as the effective toughness. We then investigate numerically the impact of the inclusion toughness on the effective fracture properties, stressing out the decisive influences of the material disorder as well as the interaction mechanisms. To capture theoretically both effects, we develop in Section 4 a homogenization framework that predicts the intrinsic effective toughness of disordered materials for the competing trapping and deflection mechanisms. Finally, in Section 5, the performances of the proposed homogenization model are assessed through the comparison with numerical results obtained over a broad range of microstructural parameters (inclusion toughness, density and shape).

\section{LEFM-based perturbative approach for simulations of large-scale crack propagation}

When a crack propagates in a heterogeneous material, one must envisage all possible geometric extensions before selecting the path followed during the subsequent propagation event. This specificity provides a natural advantage to perturbative approaches of Linear Elastic Fracture Mechanics. Based on Bueckner-Rice weight function theory (Bueckner, 1970; Rice, 1985), they provide local stress intensity factor variations arising from any small crack front geometrical perturbations from a reference crack without having to solve the whole elasticity problem. The numerical method recently proposed by Lebihain et al. (2020) builds on this powerful framework to model crack propagation in composite materials containing millions of inclusions. We briefly recall in this section the main ingredients of the approach.

\subsection{Heterogeneous microstructure and toughness field}

We consider a semi-infinite crack $\mathcal{F}$ embedded in an infinite periodic body. In the following, we adopt the usual convention of LEFM and note $x$, the direction of crack propagation, $y$, the direction orthogonal to the crack plane, $z$, the direction parallel to the crack front $\mathcal{F}$, and $L_{z}$, the period in the $z$-direction. At a given time t, the position of the crack front is noted $x(t)$ (Fig. 1.(a)).

The material is made of two phases: a homogeneous matrix and tough spherical or cubical inclusions. The inclusions are considered to be distributed isotropically and share the same diameter (or edge length) $d$. Two main assumptions are made regarding the mechanical behavior of 


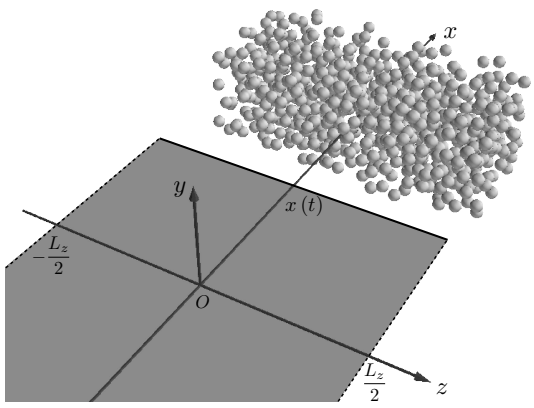

(a)

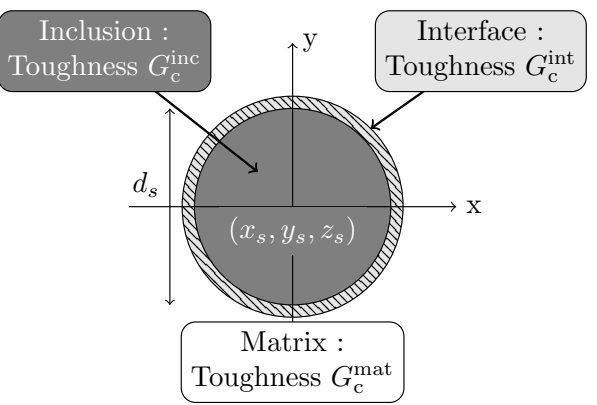

(b)

Figure 1: (a) Semi-infinite crack facing a polydisperse inclusion distribution with varying toughness; (b) Fracture properties of the inclusion, the matrix and their interface.

each phase. First, the matrix and the inclusions are assumed to be isotropically and linearly elastic and share the same Young's modulus $E$ and Poisson's ratio $v$. Second, the phases are assumed to be brittle but differ in their fracture properties: the inclusions may be tougher and/or weakly bonded to the matrix. These properties are characterized by an inclusion toughness $G_{\mathrm{c}}^{\text {inc }}$ and an interfacial toughness $G_{\mathrm{c}}^{\text {int }}$ (see Fig. 1.(b)). The assumptions underlying the proposed approach are thoroughly discussed in Lebihain et al. (2020). The description of a typical microstructure of a heterogeneous brittle solid leads to a three-dimensional toughness field $G_{\mathrm{c}}(z, x, y)$.

\subsection{Calculation of the SIFs along the crack front}

Macroscopic tensile loading.

We consider a semi-infinite plane crack in a fracture specimen loaded under tension (Mode I) at a constant opening rate $\dot{\delta}$ (see Fig. 1). The effect of both the loading conditions $\delta$ and the sample geometry are included in the proposed model via the evolution of the macroscopic Energy Release Rate (ERR) $G^{\infty}$ with the time $t$ and the crack position $x(t)$. Following (Ponson and Bonamy, 2010), $G^{\infty}$ reads at first-order :

$$
G^{\infty}(t)=G_{0}\left(1+\frac{v_{\mathrm{m}} t-x(t)}{\mathcal{L}}\right)
$$

where $G_{0}=G^{\infty}\left(\delta_{0}, x=0\right)$ is the loading for an initial opening $\delta_{0}$ at $x=0$ and $t=0$. The structural length $\mathcal{L}$ and the driving velocity $v_{\mathrm{m}}$ are defined by:

$$
\mathcal{L}=-G_{0} /\left.\frac{\partial G^{\infty}}{\partial x}\right|_{\delta_{0}, 0} ; v_{\mathrm{m}}=-\left.\dot{\delta} \frac{\partial G^{\infty}}{\partial \delta}\right|_{\delta_{0}, 0} /\left.\frac{\partial G^{\infty}}{\partial x}\right|_{\delta_{0}, 0} .
$$

Both $\mathcal{L}$ and $v_{\mathrm{m}}$ are prescribed parameters in the performed simulations. The structural length scale $\mathcal{L}$ is related to the specimen geometry and the loading conditions ${ }^{1}$, and controls the evolution of the macroscopic ERR $G^{\infty}$ as the crack advances, while $v_{\mathrm{m}}$ corresponds to the average (in time) crack velocity.

\footnotetext{
${ }^{1} \mathcal{L}$ is often found on the order of the specimen size.
} 


\section{Perturbed stress intensity factors.}

In a homogeneous material, the semi-infinite crack would undergo stable coplanar propagation at the speed $v_{\mathrm{m}}$, and the crack front $\mathcal{F}$ would remain straight at the instantaneous position $x(t)=v_{\mathrm{m}} t$. But material heterogeneities distort the crack front both within the mean fracture plane (crack trapping) and out of it (crack deflection). In the following, we note $f_{x}(z, t)$ the in-plane perturbation of the crack front, and $f_{y}(z, t)$ its out-of-plane perturbation. The in-plane perturbation is defined from the reference crack position $x(t)$ (see Fig. 2) so that it satisfies the condition $\left\langle f_{x}(z, t)\right\rangle_{z}=0$.

Assuming quasi-static crack propagation, one can use the formulæ of Gao and Rice (1986) (for the in-plane perturbation of the crack front) and those of Movchan et al. (1998) (for the outof-plane perturbation of the crack surface) to compute the perturbed SIFs $\left(K_{p}\right)_{p \in\{\text { IIIIIIII }\}}$ from the reference geometry. At first order in $f_{x}$ and $f_{y}$, the expressions of the perturbed SIFs read:

$$
\left\{\begin{array}{l}
\frac{K_{\mathrm{I}}(z, t)}{K_{\mathrm{I}}^{\infty}(t)}=1-\frac{f_{x}(z, t)}{2 \mathcal{L}}-\frac{1}{2 \pi} \mathrm{PV} \int_{-\infty}^{+\infty} \frac{f_{x}(z, t)-f_{x}\left(z^{\prime}, t\right)}{\left(z-z^{\prime}\right)^{2}} d z^{\prime} \\
\frac{K_{\mathrm{II}}(z, t)}{K_{\mathrm{I}}^{\infty}(t)}=\frac{1}{2} \frac{\partial f_{y}}{\partial x}(z, t)+\frac{2-3 v}{2-v} \frac{1}{2 \pi} \mathrm{PV} \int_{-\infty}^{+\infty} \frac{f_{y}(z, t)-f_{y}\left(z^{\prime}, t\right)}{\left(z-z^{\prime}\right)^{2}} d z^{\prime} \\
\frac{K_{\mathrm{III}}(z, t)}{K_{\mathrm{I}}^{\infty}(t)}=-\frac{2(1-v)^{2}}{2-v} \frac{\partial f_{y}}{\partial z}(z, t)
\end{array}\right.
$$

where $K_{\mathrm{I}}^{\infty}(t)=\sqrt{\frac{E}{1-v^{2}} G^{\infty}(t)}$ denotes the macroscopic mode I SIF related to the unperturbed geometry and the symbol PV a Cauchy principal value.

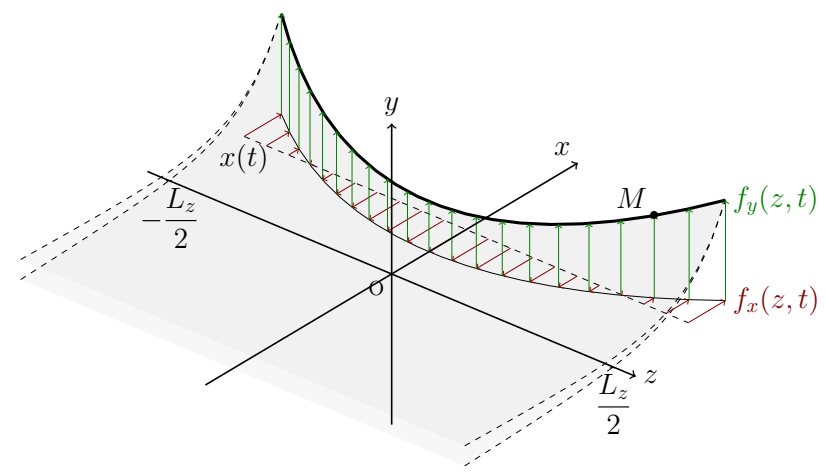

Figure 2: Geometrical perturbations of a semi-infinite planar crack located at $x(t) . f_{x}(z, t)$ and $f_{y}(z, t)$ represent its in-plane and out-of-plane perturbations, respectively.

Formulæ (3) permit to compute the SIFs, and thus the ERR $G$ at any location $M$ along the crack front. Since the toughness values along the front and in its vicinity are determined from the position $\left(z, x(t)+f_{x}(z, t), f_{y}(z, t)\right)$, there remains only one missing piece in the puzzle, the propagation criterion, which is detailed hereafter. 


\subsection{Propagation criterion}

The proposed propagation criterion combines a direction criterion, the Generalized Maximum Energy Release Rate criterion, and a kinetic law that consists in a viscous regularization of Griffith's criterion, that are detailed below.

\section{Direction criterion.}

Crack propagation is generally considered to occur within the plane orthogonal to the local tangent to the crack front. Here, we consider instead that each point $\mathrm{M}$ on the crack front propagates within the $(x M y)$ plane. The errors introduced are of second-order and thus negligible within the proposed first-order perturbation model.

The propagation direction $\theta$ selected by the crack within the $(x M y)$ plane (see Fig. 3.(a)) is predicted by the Generalized Maximum Energy Release Rate (GMERR) criterion (He and Hutchinson, 1989; Gurtin and Podio-Guidugli, 1998).

Propagation occurs in the direction $\theta$ such that $\left(G-G_{\mathrm{c}}\right)(\theta)$ be maximal.

This criterion has been shown to successfully predict crack trajectories in tearing tests of brittle polymeric thin sheets with weakly (Ibarra et al., 2016) and strongly (Takei et al., 2013) anisotropic fracture properties.

Note that the GMERR criterion reduces to the standard MERR criterion when the crack is propagating in a homogeneous phase, i.e. in the matrix or the inclusions. When the crack is on the interface between the matrix and an inclusion (see Fig. 3.(a)), the knowledge of the angular distributions of $G_{\mathrm{c}}$ and $G$ is required to predict the subsequent propagation direction $\theta$. While the former is given by the knowledge of the microstructure introduced in Section 2.1, the latter can be computed from the perturbed stress intensity factors of Eq. (3).

Let us first consider a point $\mathrm{M}$ on a crack front which has just landed on an inclusion with an attack angle $\theta_{\text {ini }}$ at a landing height $y_{\text {landing }}$ corresponding to a tangent angle $\theta_{\tan }$, as depicted in Fig. 3.(a). The angular distribution of $G$ can be deduced combining Amestoy-Leblond's formulæ (Amestoy and Leblond, 1992; Leblond, 1999), that link local stress intensity factors just after an arbitrary kink to those just before it, with Irwin's formula (Irwin, 1962). It reads:

$$
\begin{aligned}
& G(\theta)=\frac{1-v^{2}}{E}\left(K_{\mathrm{I}}^{2}(\theta)+K_{\mathrm{II}}^{2}(\theta)\right), \text { where } \quad K_{\mathrm{I}}(\theta)=F_{\mathrm{I}, \mathrm{I}}(\alpha) K_{\mathrm{I}}(z, t)+F_{\mathrm{I}, \mathrm{II}}(\alpha) K_{\mathrm{II}}(z, t) \\
& K_{\mathrm{II}}(\theta)=F_{\mathrm{II}, \mathrm{I}}(\alpha) K_{\mathrm{I}}(z, t)+F_{\mathrm{II}, \mathrm{II}}(\alpha) K_{\mathrm{II}}(z, t)
\end{aligned}
$$

where the $\left(F_{i, j}\right)$ are universal functions which depend only on the kink angle $\alpha=\theta-\theta_{\text {ini }}$. In the numerical procedure, only first-order terms of Eq. 5 are accounted for.

An example of the GMERR criterion is plotted in Fig. 3.(b). The GMERR criterion allows to predict if the crack will propagate in the tangent direction $\theta_{\tan }$ and by-pass the inclusion, or along the direction $\theta_{\max }$ predicted by the classical MERR criterion, and cross the inclusion.

\section{Kinetic law.}




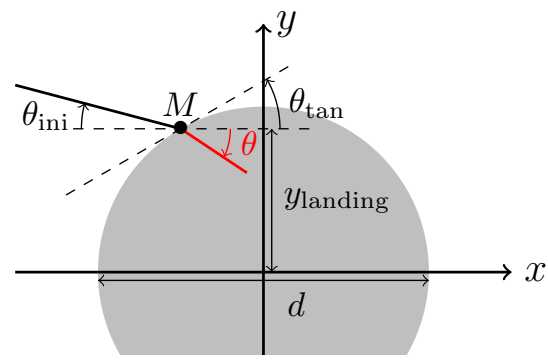

(a)

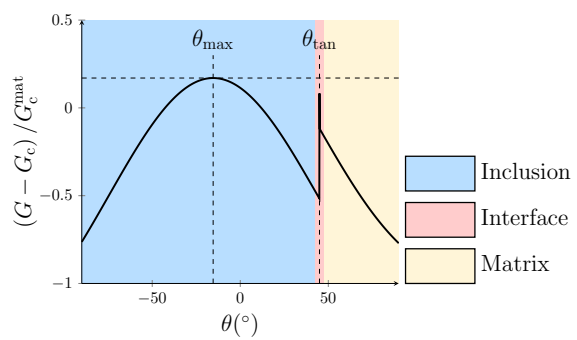

(b)

Figure 3: Application of the GMERR criterion for (a) a crack landing at a height $y_{\text {landing }}=0.25 d$ with an angle $\theta_{\text {ini }}$ on an inclusion characterized by its toughness $G_{\mathrm{c}}^{\text {inc }}$ and the interface toughness $G_{\mathrm{c}}^{\text {int }}$. (b) Associated angular distribution of

$G-G_{\mathrm{c}}$ which displays two preferential directions $\theta_{\max }$ for the crossing of the inclusion and $\theta_{\tan }$ for its by-pass.

The last missing ingredient is the kinetic law that relates the local crack velocity $v$ to $G$ and $G_{\mathrm{c}}$. For brittle materials, this kinetic law can be derived from Griffith (1921)'s criterion by accounting for the variations of the toughness with crack speed (Kolvin et al., 2015; Chopin et al., 2018). It reads :

$$
G=G_{\mathrm{c}}(v)=G_{\mathrm{c}}\left(v_{\mathrm{m}}\right)\left(1+\frac{v-v_{\mathrm{m}}}{v_{0}}\right) \quad(\text { for } v>0) \quad \Leftrightarrow \quad v=\left[v_{\mathrm{m}}+v_{0} \frac{G-G_{\mathrm{c}}\left(v_{\mathrm{m}}\right)}{G_{\mathrm{c}}\left(v_{\mathrm{m}}\right)}\right]^{+}
$$

where $v_{0}=G_{\mathrm{c}}\left(v_{\mathrm{m}}\right) /\left.\frac{\partial G_{\mathrm{c}}}{\partial v}\right|_{v_{\mathrm{m}}}$ is a characteristic velocity of the material tied to the rate-dependency of its toughness, and $[\cdot]^{+}$the positive part function. This equation of motion has been largely used in the literature (see for example (Gao and Rice, 1989; Ramanathan et al., 1997; Ponson and Bonamy, 2010)) and was recently shown to capture quantitatively the relaxation dynamics of a crack depinning from a single obstacle (Chopin et al., 2018).

\subsection{From periodic to disordered systems}

The numerical method is summarized briefly in Appendix A. It builds on analytical expressions of the SIFs, allowing an efficient computation of quantities of interest like the ERR from the discretization of the front only. As a result, crack propagation in heterogeneous media including as many as several million inclusions can be computed in only a few hours using a single core computer. With such performances, 3D fracture simulations of very large specimens can be achieved, as illustrated in Fig. 4. It allows for the description of the interaction of a crack with heterogeneities through two mechanisms : crack trapping, where the crack is pinned by tough inclusions and bows in-plane between the heterogeneities, and crack deflection, where the crack by-passes tough inclusions by propagating out-of-plane (see Fig. 4.(a)). We observe in Fig. 4.(b) out-of-plane excursions of the crack that develop at a scale far larger than the heterogeneity size. Since the pioneering work of Mandelbrot et al. (1984), it is well-known that fracture surfaces display a unique scaling behavior referred to as self-affinity. The careful study of the multiscale structure of the surface roughness is left for future work.

In this study, we focus on the effective fracture properties of brittle composite.Lebihain et al. (2020) investigated the influence of the fracture properties of the inclusions and their shape on 
the mechanism selection and its ultimate impact on the effective toughness, considering periodic arrangements of tough inclusions. We tackle here the difficult question of the effective toughness of disordered brittle solids both numerically in Section 3 and theoretically in Section 4.

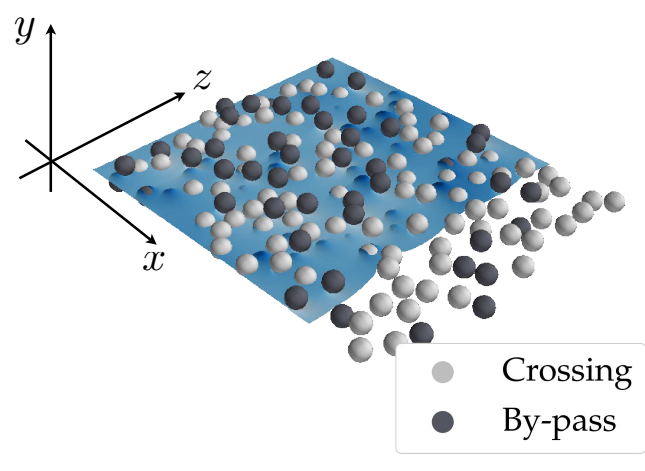

(a)

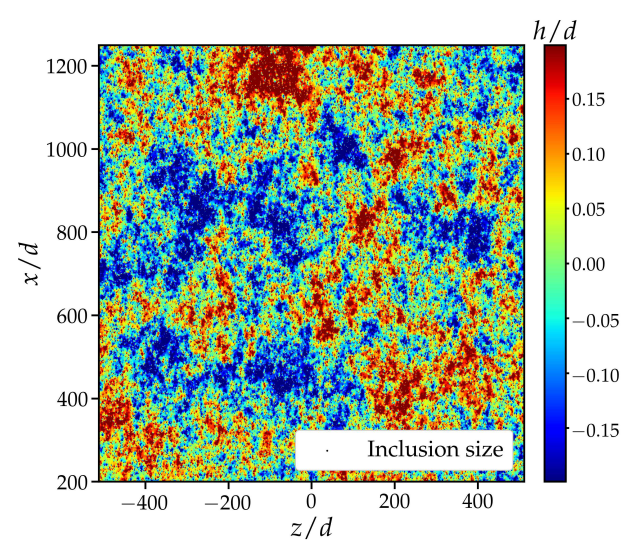

(b)

Figure 4: 3D fracture simulations of large specimens with disordered microstructures illustrating the computational performance of the newly developed numerical method: (a) propagation through a matrix containing randomly distributed tough inclusions that have been either by-passed (dark grey) or crossed (light grey); (b) fracture surface topography $h(x, y)$ normalized by the inclusion diameter $d$, resulting from the interaction of the crack with about $10^{6}$ tough inclusions. The computation takes less than one hour on a single core computer.

\section{Intrinsic fracture properties of three-dimensional heterogeneous materials}

When a crack propagates in a composite material, it interacts with material heterogeneities. These heterogeneities may pin the crack front, thus requiring the macroscopic loading to increase to permit further crack propagation. The prediction of this increased crack growth resistance is far from being trivial for a coplanar crack interacting with a disordered distribution of tough heterogeneities (Roux et al., 2003; Roux and Hild, 2008; Patinet et al., 2013b; Démery et al., $2014 a, b)$. In this case, the crack front may indeed interact with a large number of obstacles simultaneously, obliging the front to collectively escape from them to ensure propagation. While these works provide valuable insights into the influence of the material disorder on fracture properties, they have overlooked the strong impact of the local interaction mechanisms between the crack front and the heterogenities on the homogenized fracture properties. On the contrary Gao and Rice (1989), Bower and Ortiz (1991), Hossain et al. (2014) and Brach et al. (2019) have studied these mechanisms in great details, and show how they could impact the overall crack growth resistance. But their study was limited to two-dimensional or periodic microstructures so that they did not address the role of collective depinning of the crack from obstacles on the macroscopic toughness. Here, we show that both effects are relevant and should be carefully captured to predict the effective fracture properties of disordered solids. To illustrate this idea, we study crack growth in three-dimensional materials embedding a random distribution of tough inclusions for which a competition between crossing and by-pass mechanisms take place. To define an effective toughness, we first explore in Section 3.1 the various definitions found in 
the literature. We then describe in Section 3.2 the scale-separation condition under which those definitions converge to a unique value that can be considered as the intrinsic effective toughness of the composite material. The influence of the inclusion toughness on the effective toughness is finally investigated numerically in Section 3.3, stressing out the decisive influence of both the material disorder and the local interaction mechanisms on the effective fracture properties.

\subsection{The effective toughness : three possible definitions for a single material property}

Three possible definitions of the effective toughness $G_{\mathrm{c}}^{\mathrm{eff}}$ can be found in the literature:

1. The maximum energy release rate imposed by the loading during crack propagation $G_{\max }^{\infty}$. This definition was adopted by Hossain et al. (2014) and Brach et al. (2019) in twodimensional phase-field simulations of the interaction of a crack with elastic and toughness heterogeneities. It was also used by Vasoya et al. (2016) to characterize the reinforcement of weak interfaces by a periodic array of tough obstacles. The loading has to be increased up to $G_{\max }^{\infty}$ to break the whole specimen.

2. The average energy release rate imposed by the loading during crack propagation $G_{\text {mean }}^{\infty}$. This definition was adopted by Patinet et al. (2013b) in numerical simulations of threedimensional coplanar crack propagation in disordered systems and Li and Zhou (2013) in cohesive zone model simulations of two-dimensional crack propagation in composite ceramics. It quantifies the loading level $G_{\text {mean }}^{\infty}$ at which crack propagation occurs, without necessarily leading to total failure of the structure.

3. The effective fracture energy $\left\langle G_{\mathrm{c}}^{\mathrm{frac}}\right\rangle$ defined as the average energy dissipated per unit surface during crack propagation. This definition is also explored in this work.

The numerical method presented in Section 2 allows to track the crack front position at each time step, and thus the evolution of the macroscopic ERR $G^{\infty}$ over time following Eq. (1), from which it is possible to measure both its average $G_{\text {mean }}^{\infty}$ and maximum $G_{\max }^{\infty}$ values (see Fig. 5.(a)). In Fig. 5.(a), we observe that crack propagation in disordered materials is highly intermittent (Bonamy, 2009; Barés et al., 2014), and is characterized by two different phases : pinning phases, during which the crack does not progress and the macroscopic loading increases, are separated by phases of sudden propagation, during which the loading decreases at a rate $\frac{\partial G^{\infty}}{\partial x}=-\frac{G_{0}}{\mathcal{L}}$ (see Eq. (1)). The maximum value of the macroscopic ERR $G_{\max }^{\infty}$ is set by the strongest pinning configuration, while its average value $G_{\text {mean }}^{\infty}$ is determined from all configurations the crack visits during its propagation. The latter is thus expected to strongly depend on the structural length scale $\mathcal{L}$, which controls the loading evolution during crack propagation.

It is also possible to track the energy locally dissipated by the fracture processes during numerical simulations. Indeed, local maps of dissipated surface energy $G_{\mathrm{c}}^{\text {frac }}(z, x)$ can be computed from the procedure described in Appendix B. An example of such a map is shown in Fig. 5.(b), where circular domains are characteristic of the crossing mechanism, while moon-shaped patterns can be ascribed to by-pass events. The larger surface energy patterns with a lower dissipated energy density are the results of kinetic effects during the relaxation of the crack front perturbation after leaving an inclusion (Chopin et al., 201 8).

We observe in Fig. 7 that the effective fracture energy $\left\langle G_{\mathrm{c}}^{\text {frac }}\right\rangle$, defined as the spatial average of $G_{\mathrm{c}}^{\mathrm{frac}}(z, x)$, is equal to the average energy release rate $G_{\text {mean }}^{\infty}$, although they stem from markedly different statistics (see Appendix C). This observation is far from trivial since $\left\langle G_{\mathrm{c}}^{\mathrm{frac}}\right\rangle$ is defined as the average of the field $G_{c}^{\text {frac }}$ of dissipated surface energy, which is a local quantity set by the instantaneous $\operatorname{ERR} G(z, t, \theta)$ (see Eq. 29) that varies along the distorted crack front, while $G_{\text {mean }}^{\infty}$ 

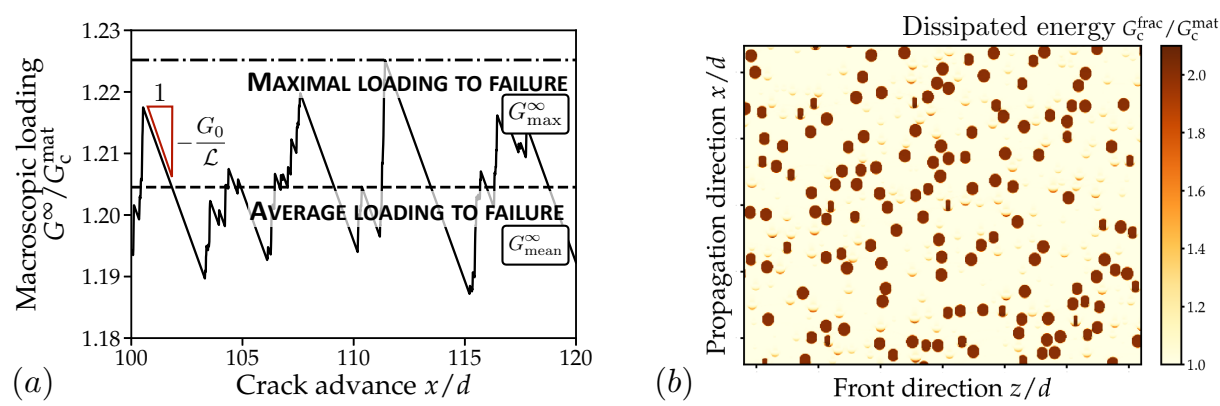

Figure 5: Definitions of the effective toughness : (a) $G_{\text {mean }}^{\infty}$ (in dashed line) and $G_{\max }^{\infty}$ (in dash-dotted line) defined respectively as the average and the maximum values of the macroscopic ERR $G^{\infty}$ imposed by the tensile loading during crack propagation. (b) $\left\langle G_{\mathrm{c}}^{\mathrm{frac}}\right\rangle$ defined as the spatial average of the fracture energy $G_{\mathrm{c}}^{\mathrm{frac}}(z, x)$ dissipated during crack propagation, computed from the methodology described in Appendix B.

is determined from $G^{\infty}$, which is a macroscopic quantity related to the far-field loading. Recent works suggest that this observation remain valid in presence of elastic heterogeneities (Dal Maso and Toader, 2002; Cagnetti et al., 2019; Schneider, 2020) ${ }^{2}$.

If $\left\langle G_{\mathrm{c}}^{\mathrm{frac}}\right\rangle$ and $G_{\text {mean }}^{\infty}$ are equal, they do not always coincide with the maximum energy release rate $G_{\max }^{\infty}$. In particular, the discrepancy between the average energy release rate $G_{\text {mean }}^{\infty}$ and the maximum energy release rate $G_{\max }^{\infty}$ seems to strongly depend on the structural length $\mathcal{L}$ that controls the evolution of $G^{\infty}$ (see Fig. 5).

\subsection{Towards a unified value of the effective toughness : the decisive impact of the loading conditions}

\subsubsection{Impact of the structural length $\mathcal{L}$ on the effective toughness}

In this section, we investigate the impact of the structural length $\mathcal{L}$ on the maximum energy release rate $G_{\mathrm{max}}^{\infty}$, the average energy release rate $G_{\mathrm{mean}}^{\infty}$ and the effective fracture energy $\left\langle G_{\mathrm{c}}^{\mathrm{frac}}\right\rangle$ to determine a scale-separation condition under which an intrinsic value for the effective toughness $G_{\mathrm{c}}^{\text {eff }}$ can be measured by decoupling the material problem related to $G_{\mathrm{c}}^{\text {eff }}$ from the structural one related to $G^{\infty}$.

We run numerical simulations of quasi-static crack propagation at a driving speed $v_{m}=$ $10^{-9} v_{0}$ on large disordered systems of size $L_{z} \times L_{x} \times L_{y}=256 d \times 288 d \times 16 d$ containing hundreds of thousands of inclusions. We consider a distribution of monodisperse spherical inclusions of diameter $d$ at an inclusion density $\rho_{\text {inc }}=25 \%$ for three different inclusion toughness levels: $G_{\mathrm{c}}^{\text {inc }}=1.5 G_{\mathrm{c}}^{\text {mat }}, G_{\mathrm{c}}^{\text {inc }}=2 G_{\mathrm{c}}^{\text {mat }}$ and $G_{\mathrm{c}}^{\text {inc }}=3 G_{\mathrm{c}}^{\text {mat }}$. The interface toughness is chosen equal to that of the matrix $G_{\mathrm{c}}^{\text {int }}=G_{\mathrm{c}}^{\text {mat }}$. Finally, the structural length $\mathcal{L}$, characteristic of the loading variations, is varied from $10^{-1} d$ to $10^{6} d$. The crack front is discretized with a fine mesh $\Delta z=d / 16$. The results are averaged over five inclusion distribution realizations ${ }^{3}$.

\footnotetext{
${ }^{2}$ Note that our result is valid in the quasi-static regime, when the driving velocity $v_{m}$ and the velocity $v_{0}$ characteristic of the rate-dependent fracture energy is smaller than the Rayleigh wave speed $c_{\mathrm{R}}$. Indeed, it is assumed here that all the energy available at the crack tip is dissipated through fracture processes due to the rate-dependency of the fracture energy (see Section 2.3), and not radiated within the bulk through elastic waves.

${ }^{3}$ An average over five realizations of the inclusion distribution is performed for all the numerical results presented in the following.
} 


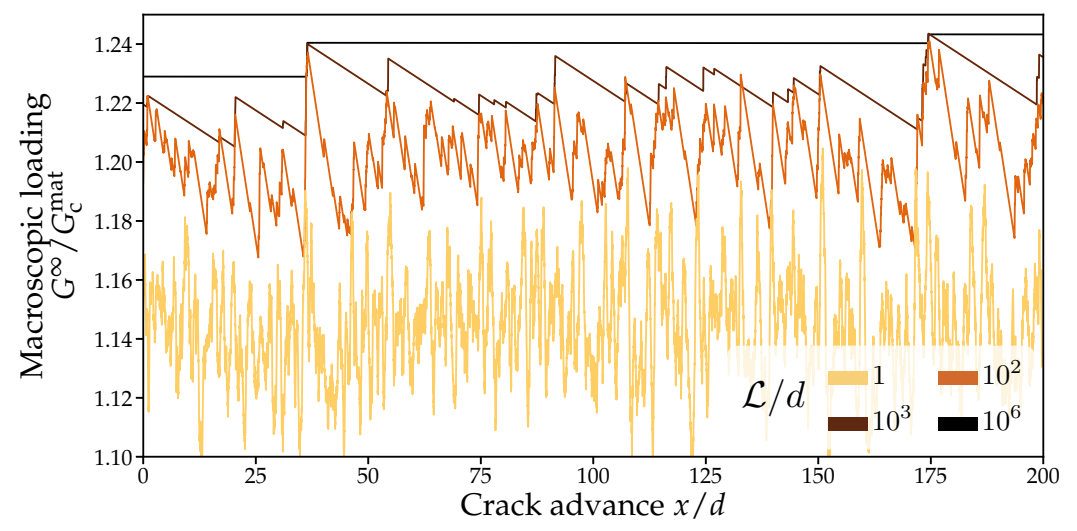

Figure 6: Impact of the structural length $\mathcal{L}$ on the evolution of the macroscopic ERR $G^{\infty}$ during crack propagation : evolution of the macroscopic ERR $G^{\infty}$ imposed by the loading while the crack is interacting through the crossing and by-pass mechanisms with a distribution of tougher inclusions of density $\rho_{\text {inc }}=25 \%$ and toughness $G_{\mathrm{c}}^{\text {inc }}=2 G_{\mathrm{c}}^{\text {mat }}$.

We plot the evolution of the macroscopic ERR $G^{\infty}$ imposed by the loading for various structural length values $\mathcal{L}$ in Fig. 6. The larger the structural length $\mathcal{L}$, the slower the decrease of $G^{\infty}$ when the crack propagates (see Eq. (1)). Thus, a large structural length $\mathcal{L} \simeq 10^{6} d$ induces a screening of almost all the subsequent stable pinning configurations. The macroscopic ERR reduces to a constant function, and $G^{\infty} \quad$ coincides with $G_{\text {mean }}^{\infty}$. For an intermediate structural length $\mathcal{L} \simeq 10^{2} d$, the crack visits a larger number of pinning configurations due to the rapid decrease of $G^{\infty}$ during crack propagation: the average value of the macroscopic loading $G_{\text {mean }}^{\infty}$ is lowered while its maximum value $G_{\max }^{\infty}$ remains unchanged since it is determined from the toughest configuration visited by the crack. When $\mathcal{L}$ approaches the size of the inclusion $d$, we observe a shift of behavior and both the average value $G_{\text {mean }}^{\infty}$ and the maximum $G_{\max }^{\infty}$ of the macroscopic ERR are reduced. The interaction between a crack and the inclusions is no more governed by long-range elastic interactions but rather by local restoring forces encapsulated in the term $-\frac{f_{x}}{\mathcal{L}}$ of Eq. (3). The macroscopic loading required to make the crack propagate is lowered due to the dominant contribution of the restoring forces. It must be emphasized that this effect is purely three-dimensional since it is due to in-plane perturbations along the crack front. Thus $G_{\mathrm{max}}^{\infty}$ is not expected to decay as $\mathcal{L}$ decreases in a two-dimensional setting.

The impact of the structural length $\mathcal{L}$ on the effective toughness $G_{\mathrm{c}}^{\text {eff }}$ is presented in Fig. 7 . Note that for $\mathcal{L}=10^{5} d$ the three possible definitions for the effective toughness converge towards a unique value, which can then be unambiguously referred to as the effective toughness $G_{\mathrm{c}}^{\mathrm{eff}}$ of the composite. Such a condition is relatively standard in the homogenization theory. However, the macroscopic length scale $\mathcal{L}$ is here set by the size of the fracture specimen and is affected by the imposed loading conditions, a feature that highlights the specificity of brittle fracture.

As foreseen by Hossain et al. (2014), the maximum energy release rate $G_{\max }^{\infty}$ appears to be, in practice, the most suitable choice to measure the intrinsic homogenized fracture properties of heterogeneous materials, as it requires smaller specimens. Indeed significant variations start to appear for $\mathcal{L} \lesssim 10^{2}-10^{3} d G_{\text {max }}^{\infty}$ while they appear for $\mathcal{L} \lesssim 10^{4}-10^{5} d$ for $G_{\text {mean }}^{\infty}$ and $\left\langle G_{\mathrm{c}}^{\text {frac }}\right\rangle$ 
(see Fig. 7). As a result, using $G_{\text {mean }}^{\infty}$ to measure the effective toughness $G_{\mathrm{c}}^{\mathrm{eff}}$ induces an error of about $20 \%$ on the reinforcement for $\mathcal{L} \simeq 10^{3} d$, while the error ranges between $30 \%$ and $40 \%$ for $\mathcal{L} \simeq 10^{2} d$.

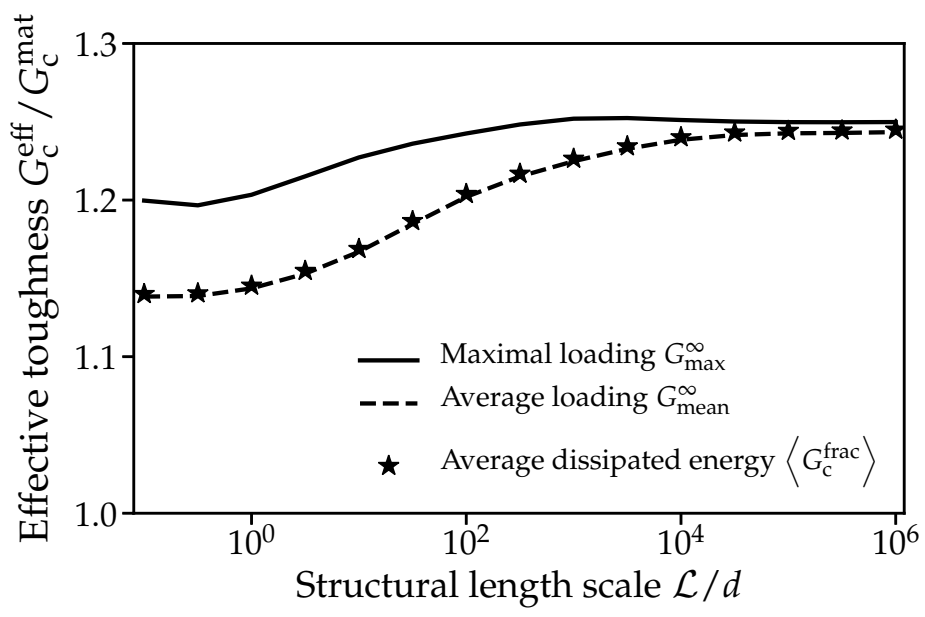

Figure 7: Impact of the structural length $\mathcal{L}$ on the possible definitions of the effective toughness $G_{\max }^{\infty}, G_{\text {mean }}^{\infty}, G_{\mathrm{c}}^{\text {frac }}$ Note that they all converge to a same value for a sufficiently large structural length $\mathcal{L} \ll 10^{5} d$.

\subsubsection{Implications on numerical simulations and experimental set-ups}

Our findings raise the question of the choice of the boundary conditions and specimen size in numerical simulations. In the proposed numerical method, the structural length $\mathcal{L}$ is a parameter of the model and can be set at will. In FEM-based computational methods, $\mathcal{L}$ is often set by loading conditions. Recent forefront numerical studies computed the effective toughness of two-dimensional heterogeneous materials from phase-field simulations using a well-thought surfing boundary condition (Hossain et al., 2014; Brach et al., 2019). This condition appears to set $\mathcal{L} \simeq L_{y}$, where $L_{y}$ is the height of the simulation domain in the direction perpendicular to crack propagation. $L_{y}$ is typically no more than one order of magnitude larger than the defect size, due to computational costs. This leaves no choice but to measure the effective toughness from the maximum energy release rate $G_{\max }^{\infty}$, an option that was indeed chosen by Hossain et al. (2014). It actually allows to predict accurately the effective toughness in two-dimensional cases since the decrease of $G_{\max }^{\infty}$ for $\mathcal{L}<10^{3} d$ only occurs in three-dimensional settings. However, it is worth noticing that such boundary conditions might lead to an underestimation of the effective toughness of disordered materials when transposed into three-dimensions.

From an experimental point of view, the average energy release rate $G_{\text {mean }}^{\infty}$ is the easiest variable to measure since it can be estimated from the total energy released during crack propagation and thus extracted from the force-displacement curve. Estimating the maximum energy release rate $G_{\max }^{\infty}$ requires to track the evolution of the macroscopic elastic energy release rate during crack propagation. The procedure is naturally more complex but can be achieved through the compliance method (Wang and Xia, 2017; Vasudevan et al., 2019) or DIC computations (Roux 
and Hild, 2006; Grabois et al., 2018). The estimation of the local map of dissipated fracture energy $G_{\mathrm{c}}^{\text {frac }}$ happens to represent a particular conundrum since it requires to track in real-time the local ERR along the crack front $G(z, t)$ as well as the crack trajectory and dynamics, which allow for the estimation of the local velocity $v(z, t)$. In the case of three-dimensional crack propagation, this might be possible but nonetheless costly since observing three-dimensional crack front configurations requires powerful imaging techniques such as in-situ micro-tomography (Renard et al., 2017; Chateau et al., 2018). Moreover, complex digital volume correlation (DVC) procedures have to be developed in order to estimate the local ERR distribution from the data brought by the imaging techniques (Lachambre et al., 2015). Finally, Eq. (B-27) supposes to be able to get all this information at very fine temporal and spatial scales. While recent progress in imaging techniques allows to get such "4D" data sets, it is unlikely that such methods will become systematic. It ultimately enforces conditions on the specimen geometry that sets the structural length $\mathcal{L}$, which is usually of the order of the specimen size (Pallares et al., 2009; Vasudevan et al., 2019). One could either ensure that the structural length is large enough $\mathcal{L} \simeq 10^{4} d$ and measure $G_{\text {mean }}^{\infty}$ from the force-displacement curve or deploy more advanced experimental techniques and extract the effective toughness from $G_{\max }^{\infty}$ for $\mathcal{L} \sim 10^{2} d$. In fracture experiments of brittle rocks, where the heterogeneity size is typically $d=100 \mu \mathrm{m}$ (Nasseri and Mohanty, 2008; Chandler et al., 2016), the latter order of magnitude is easily satisfied for a standard $10 \mathrm{~cm}$-size sample while the former is often violated since it would require a $3 \mathrm{~m}$ large specimen ${ }^{4}$. This remark drives the need for the spreading of advanced experimental methods for fracture properties measurements such as DIC techniques. Otherwise, experimental measurements might lead to an underestimation of the effective fracture properties and to size-effect.

\subsection{Effective toughness of disordered composite materials}

In the remainder of this study, we set the structural length $\mathcal{L}=10^{6} d$ to fully decouple the effective toughness measurements from the structural problem. Crack propagation is made to occur over a distance $L_{x}=L_{z}$ to ensure that the toughest pinning configuration is visited Kolton et al. (2013). Variables of interest (front position, local maps of effective fracture energy, etc.) are recorded after a propagation length $L^{*}=\sqrt{L_{z} d}$ to reach a stationary regime independent of the initial planar configuration (Patinet et al., 2013b). Under these assumptions, the effective toughness $G_{\mathrm{c}}^{\text {eff }}$ can be measured through the evaluation of the maximum value of the macroscopic ERR imposed by the loading $G_{\max }^{\infty}$ :

$$
G_{\mathrm{c}}^{\mathrm{eff}}=\max _{x \in\left[0, L_{x}\right]} G^{\infty}(x)
$$

We now investigate the impact of microstructural features on effective fracture properties and start by studying the influence of the inclusion toughness on the effective toughness. We consider the case of a single crack propagating in large disordered systems of size $L_{z} \times L_{x} \times L_{y}=$ $256 d \times 288 d \times 16 d$, which is large enough to ensure convergence of the results with the system size (see Appendix D).

Monodisperse distributions of spherical inclusions of diameter $d$ are considered for an inclusion density $\rho_{\text {inc }}=25 \%$. The inclusion toughness varies from $G_{\mathrm{c}}^{\text {inc }}=G_{\mathrm{c}}^{\text {mat }}$ to $G_{\mathrm{c}}^{\text {inc }}=4.5 G_{\mathrm{c}}^{\text {mat5 }}$,

${ }^{4}$ For a TDCB specimen, the structural length scale $\mathcal{L}$ is on the order of one third of the specimen size.

${ }^{5}$ Note that only tougher inclusions are considered in order to promote both the crossing and the by-pass mechanisms of interaction. Weaker inclusions are always crossed, and the problem reduces to the well-understood case of coplanar crack propagation. 
which remains within the range of validity of the perturbative approach (Gao and Rice, 1989; Lebihain et al., 2020). The interface shares the fracture properties of the matrix $G_{\mathrm{c}}^{\mathrm{int}}=G_{\mathrm{c}}^{\mathrm{mat}}$. Examples of the distributions considered are given in Fig. 8.(a-c).
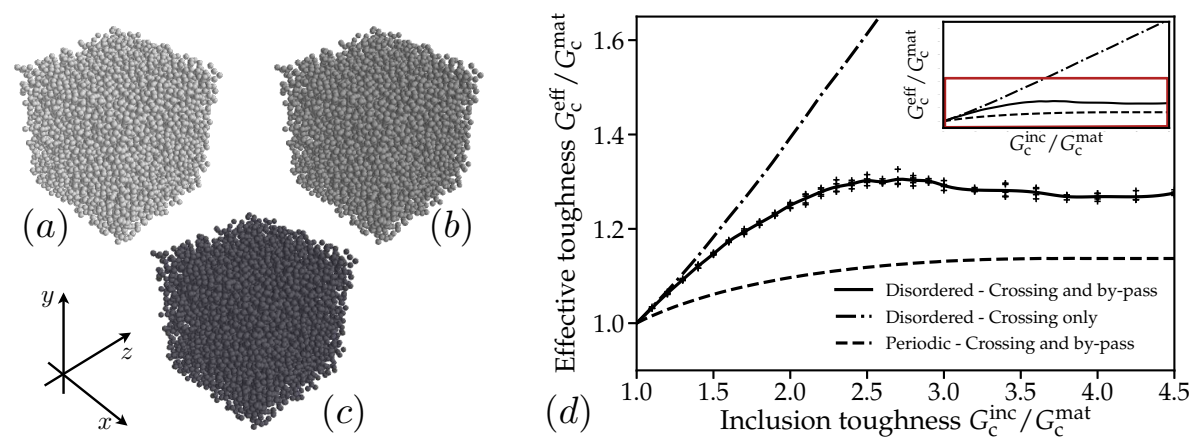

Figure 8: Impact of the inclusion toughness $G_{\mathrm{c}}^{\mathrm{inc}}$ on the effective toughness $G_{\mathrm{c}}^{\text {eff }}:(\mathrm{a}-\mathrm{c})$ the crack interacts with large distributions of spherical inclusions with varying toughness. (d) The effective toughness is estimated from the crack evolution following Eq. (7). Numerical estimates (in solid line) are compared to numerical results of coplanar simulations where the crack can interact through the sole crossing mechanism (in dash-dotted line) and periodic simulations of (Lebihain et al., 2020) taking into account both inclusion crossing and by-pass (in dashed line).

The evolution of the effective toughness with the inclusion toughness is predicted following Eq. (7) from 135 simulations in which the crack front interacts through the crossing and by-pass mechanisms with hundreds of thousands of tough inclusions. The results are plotted in Fig. 8, where averaged results are plotted in solid lines while individual simulation points are plotted with cross markers. Numerical results of three-dimensional crack propagation in disordered materials are compared to the ones of coplanar propagation, where the crack interacts with a disordered distribution of tough inclusions through the sole crossing mechanism, as well as periodic ones, where both mechanisms are modeled ${ }^{6}$. A convergence study with the front mesh size $\Delta z$ is reported in Appendix E.

From Fig. 8, we notice that:

- first, the introduction of inclusion by-pass appears to significantly limit material reinforcement by tough inclusions. Such features have already been highlighted in the periodic case (Lebihain et al., 2020) and stress out the major influence of the interaction mechanisms on the effective fracture properties;

- second, the periodic problem does not provide a proper estimate of the effective toughness of disordered materials. The material disorder seems to play a decisive role in the ultimate material reinforcement.

Designing brittle composites with optimized fracture properties requires develop a homogenization framework that rationalizes the influence of microstructural parameters such as the inclusion toughness on the effective toughness. This framework must take into account the strong

\footnotetext{
${ }^{6}$ The periodic results on the effective toughness are inferred from the work of Lebihain et al. (2020) as the average
} dissipation for multiple landing heights $y_{\text {landing }}$. 
impact of both the material disorder and the interaction mechanisms on the ultimate material reinforcement. Section 4 is dedicated to the construction of such a theoretical framework.

\section{Homogenization framework for the fracture properties of disordered brittle materials}

This section is dedicated to the construction of a semi-analytical framework for the homogenization of the effective fracture properties, accounting for the competition between several mechanisms of interaction between a crack and randomly distributed of tough inclusions. It builds on the results of Démery et al. (2014b) who developed a theoretical framework for the homogenization of fracture properties in the case of coplanar propagation, where the toughness field the crack effectively visits corresponds to the local toughness properties of the material. The method developed here generalizes this framework by taking into account the respective contributions of each interaction mechanism on the overall toughness. The proposed homogenization method is applied to three-dimensional disordered composites where two types of local interaction mechanisms are in competition, namely crossing and by-pass. The efficiency of the approach is then tested through its ability to capture the impact of the inclusion toughness on the effective toughness that has been computed through direct simulations in Fig. 8.

\subsection{Outline of the homogenization procedure}

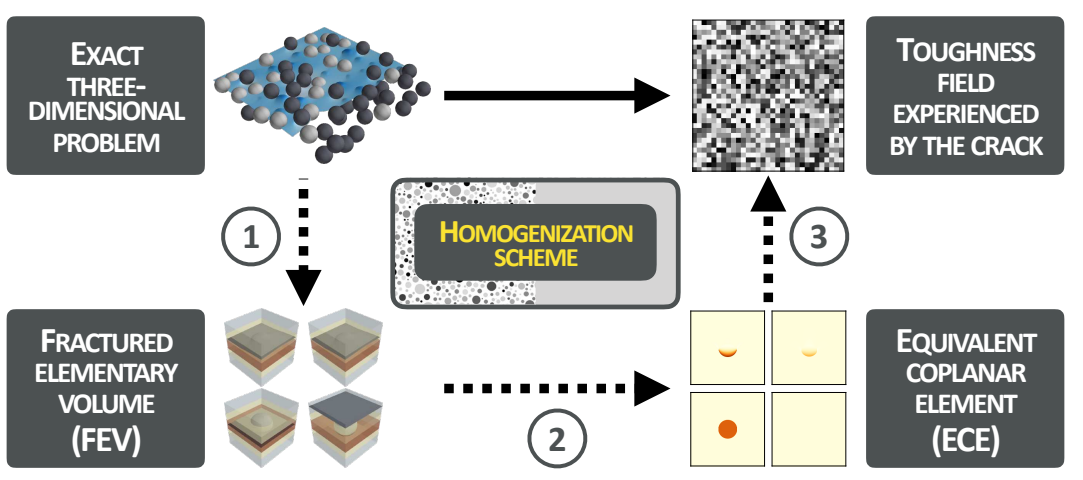

Figure 9: Three-step homogenization technique developed to predict effective toughness properties in a three-dimensional non-coplanar setting.

Démery et al. (2014b) developed a theoretical framework to predict the effective toughness from the statistical features of the local toughness field $G_{\mathrm{c}}(z, x)$, namely its average toughness $\left\langle G_{\mathrm{c}}\right\rangle$, its standard deviation $\sigma$, and its correlation lengths $\xi_{z}, \xi_{x}$. This framework has been developed in the case of coplanar crack propagation where the crack can only interact with the material disorder through the crossing mechanism. Yet, we notice in Fig. 4 that the out-of-plane deviations of the crack remain relatively small so that crack propagation is not far from planar. A homogenization scheme is then adapted from Démery et al. (2014b) to tackle the case of noncoplanar propagation through an equivalent coplanar problem.

Yet, the statistical features of the equivalent coplanar distribution of toughness $G_{\mathrm{c}}^{\text {cop }}(z, x)$ cannot be directly inferred from the three-dimensional distribution of toughness $G_{\mathrm{c}}(z, x, y)$. Indeed, 
the interaction mechanism selected by the crack governs the local toughness the crack effectively visits during propagation: if the inclusion is crossed, the crack visits the inclusion toughness whereas if the inclusion is by-passed it sees that of the interface. The determination of the equivalent coplanar problem should then derive from the actual crack-tip interaction mechanisms at play in the original fully three-dimensional problem.

Inspired by standard micromechanical models, we propose a three-step homogenization scheme:

1. First, we break down the full problem of the propagation of a crack in a disordered distribution of inclusions into multiple simpler problems, referred to as fractured elementary volumes (FEVs) problems, considering all the possible ways a crack can interact with a single inclusion and their respective probabilities. This decomposition relies on the absence of interaction during the out-of-plane by-pass of neighboring inclusions. We refer to this as the non-collective mechanisms hypothesis.

2. Second, the three-dimensional cell problems provide equivalent coplanar ones, called equivalent coplanar elements (ECEs), which derive from the way the crack interacts with the inclusion (crossing or by-pass).

3. Third, the assemblage of all the coplanar cell problems allows to get back to an equivalent coplanar toughness distribution, from which the statistical features $\left(\left\langle G_{\mathrm{c}}\right\rangle, \sigma, \xi_{z}, \xi_{x}\right)$ can be inferred under the ergodic assumption. It ultimately allows to estimate the effective toughness of three-dimensional heterogeneous brittle materials using the coplanar theory of Démery et al. (2014b).

The procedure is summarized in Fig. 9.

\subsection{The influence of material disorder: an insight from statistical physics}

We first recall the results of Démery et al. (2014a) and Démery et al. (2014b) on coplanar crack propagation in heterogeneous materials exhibiting toughness heterogeneities. Let us consider a heterogeneous plane described by its toughness field:

$$
G_{\mathrm{c}}(z, x)=\left\langle G_{\mathrm{c}}\right\rangle+\sigma g_{\mathrm{c}}(z, x)
$$

where $\left\langle G_{\mathrm{c}}\right\rangle$ is its spatial average, $\sigma$ its standard deviation and $g_{\mathrm{c}}(z, x)$, the disorder function, a dimensionless spatial field of unit variance and zero mean value.

At first-order the coplanar equation of motion reads (Démery et al., 2014b):

$$
\begin{aligned}
\frac{1}{v_{0}} \frac{\partial f_{x}}{\partial t}(z, t)= & \frac{v_{m}}{v_{0}}+\frac{G^{\infty}}{\left\langle G_{\mathrm{c}}\right\rangle}\left(1-\frac{1}{\mathcal{L}} f_{x}(z, t)-\frac{1}{\pi} \mathrm{PV} \int_{-\infty}^{+\infty} \frac{f_{x}(z, t)-f_{x}\left(z^{\prime}, t\right)}{\left(z-z^{\prime}\right)^{2}} d z^{\prime}\right) \\
& -1-\frac{\sigma}{\left\langle G_{\mathrm{c}}\right\rangle} g_{\mathrm{c}}\left(z, x=f_{x}(z, t)\right)
\end{aligned}
$$

Démery et al. (2014a) solved Eq.(9) building on an approach inspired by statistical physics (Larkin and Ovchinnikov, 1979) to predict the effective toughness of disordered material in the case of coplanar crack propagation. They proved that the effective toughness $G_{\mathrm{c}}^{\mathrm{eff}}$ relates to the statistical features of the toughness field $G_{\mathrm{c}}(z, x)$ through the following equation (Démery et al., 
2014b):

$$
\left\{\begin{array}{lr}
G_{\mathrm{c}}^{\mathrm{eff}}=\left\langle G_{\mathrm{c}}\right\rangle+\frac{\sigma^{2}}{\left\langle G_{\mathrm{c}}\right.} \frac{\xi_{z}}{\xi_{x}} & \text { if } \xi_{z} \leq L_{c} \leq L_{z} \text { (Collective pinning regime) } \\
G_{\mathrm{c}}^{\mathrm{eff}} \geq\left\langle G_{\mathrm{c}}\right\rangle+\sigma & \text { if } L_{c} \leq \xi_{z} \text { (Individual pinning regime) } \\
G_{\mathrm{c}}^{\mathrm{eff}}=\left\langle G_{\mathrm{c}}\right\rangle+\sigma \sqrt{\frac{\xi_{z}}{L_{z}}} & \text { if } L_{c} \geq L_{z} \text { (Size-dependent regime) }
\end{array}\right.
$$

where $\xi_{z}$ and $\xi_{x}$ are the correlation lengths of the local toughness in the front direction $(O z)$ and the propagation direction $(O x)$, respectively. $L_{c}=\left(\frac{\left\langle G_{\mathrm{c}}\right\rangle}{\sigma}\right)^{2} \frac{\xi_{x}^{2}}{\xi_{z}}$ is the so-called Larkin length that relates to the amplitude of the in-plane perturbation through the relation $\Delta f_{x}\left(\Delta z=L_{c}\right)=\xi_{x}$, where $\Delta f_{x}(\Delta z)=\left\langle\left[f_{x}(z+\Delta z, x)-f_{x}(z, x)\right]^{2}\right\rangle^{1 / 2}$ is the correlation function of the in-plane front perturbation. It then provides the characteristic length scale along the front direction over which the front perturbations become comparable to the inclusion size (Larkin and Ovchinnikov, 1979). From a mathematical standpoint, the disorder term $g_{\mathrm{c}}\left(z, x=f_{x}(z, t)\right)$ in the equation of motion of the crack (Eq. (9)) then cannot be linearized anymore, and the problem becomes strongly nonlinear.

Equation (10) relates the effective toughness to the statistical features of the toughness field for three different regimes of propagation:

- when $\xi_{z} \leq L_{c} \leq L_{z}$, the crack propagates in a collective pinning regime. The effective toughness is governed by the distribution of toughness visited by portions of the crack front of size $L_{c}$, called "Larkin domains". An additional toughening $\frac{\sigma^{2}}{\left\langle G_{c}\right.} \frac{\xi_{z}}{\xi_{\varepsilon}}$ then emerges from the material disorder $\sigma$ when compared to the periodic situation $G_{\mathrm{c}}^{\text {eff }}=\left\langle G_{\mathrm{c}}\right\rangle$ (Gao and Rice, 1989). This toughening arises from the competition between the elasticity of the crack that tends to maintain the front as smooth as possible and the material disorder that on the contrary tends to roughen it. Roughly speaking, the crack front ends up to get stuck by the toughest inclusions so it effectively visits regions of the local toughness field that are tougher than the average value $\left\langle G_{\mathrm{c}}\right\rangle$;

- when $L_{c}<\xi_{z}$, the crack propagates in an individual pinning regime. A Larkin domain only "sees" one defect and the effective toughness is set by the toughest inclusions so that $\left\langle G_{\mathrm{c}}\right\rangle+\sigma$ only represents a lower bound of $G_{\mathrm{c}}^{\text {eff }}$;

- when $L_{c}>L_{z}$, the effective toughness depends on the size of the system and an additional toughening emerges from finite size effects. This effect is thoroughly investigated in Appendix D.

The effective toughness displays a soft cross-over between the collective regime $L_{c}>\xi_{z}$ and the individual pinning regime $L_{c}<\xi_{z}$. Thus, in the following, the effective toughness is not strictly predicted from Eq. (10) but also takes into account the cross-over regime by interpolating the numerical data of Démery et al. (2014b). Such a soft cross-over also separates the collective regime from the size-dependent one (see Appendix D) but we do not take it into account in this study, given that the system sizes $L_{z}$ considered are systematically larger than the Larkin length $L_{c}$. 
Note that in Eq.(8), $G_{\mathrm{c}}(z, x)$ corresponds to the toughness field of the fracture plane that, in coplanar problems, corresponds to the toughness field actually visited by the crack. For threedimensionnal fracture problems where the crack is able to wander out-of-plane and alternatively visits the toughness of the matrix, the inclusion or the matrix/inclusion interface, the visited fracture field $G_{\mathrm{c}}^{\mathrm{cop}}(z, x)$ does not correspond anymore to any material plane. A comprehensive homogenization framework should then contain appropriate tools to estimate the toughness distribution actually visited by the crack, from which one can infer the statistical parameters $\left\langle G_{\mathrm{c}}\right\rangle, \sigma, \xi_{z}, \xi_{x}$ required to predict the effective toughness. The next to sections are devoted to this issue.

\subsection{The influence of the microstructural features: the fractured elementary volume}

The first step of the proposed method consists in breaking down the original non-coplanar problem into multiple cell problems called fractured elementary volumes (FEVs), where a crack interacts with a single inclusion only.

In the most general case, the composite material is made of a homogeneous matrix and an isotropic distribution of spherical inclusions $\mathcal{S}=\left(\mathcal{S}_{i}\right)$ of density $\rho_{\text {inc }}$. The diameter $\left(d_{\text {inc }, i}\right)$ of the inclusions $\left(\mathcal{S}_{i}\right)$, follows a distribution characterized by its probability density function $p_{d}$. In the same manner, the toughness $\left(G_{c, i}^{\text {inc }}\right)$ of the inclusions and the toughness $\left(G_{c, i}^{\text {int }}\right)$ of their interface follow distributions described by the respective probability density functions $p_{\text {inc }}$ and $p_{\text {int }}$. Such distributions can either be prescribed as assumed here, or result from a statistical analysis based on experimental measurements.

We call elementary volume (EV) the cell $\omega$ containing a single inclusion of diameter $d_{\text {inc }}$, toughness $G_{\mathrm{c}}^{\text {inc }}$, and interface toughness $G_{\mathrm{c}}^{\text {int }}$ embedded at the center of a cube made of the matrix material, whose edge length $L_{\rho}$ is given by:

$$
L_{\rho}=\left(\frac{\pi}{6 \rho_{\mathrm{inc}}}\right)^{\frac{1}{3}} d_{\mathrm{inc}}
$$

so that the inclusion density inside the spherical EV is equal to $\rho_{\text {inc }}$.

$\Omega$ is the statistical ensemble of possible realizations of such EVs. To the ensemble $\Omega$ is associated a probability density function $p_{\mathrm{Ev}}$. Given that the inclusion diameter and fracture properties are assumed to be independent variables, it reads:

$$
p_{\mathrm{EV}}(\omega)=p_{d}\left(d_{\text {inc }}\right) \cdot p_{\text {inc }}\left(G_{\mathrm{c}}^{\mathrm{inc}}\right) \cdot p_{\text {int }}\left(G_{\mathrm{c}}^{\mathrm{int}}\right)
$$

The interaction of the microstructural elementary volume with an incoming half-plane crack represents a realization $\omega_{\mathrm{F}}$ of a fractured elementary volume (FEV) (see Fig. 10.(a)). This interaction is described by the height $y$, at which the crack penetrates the EV. Given that the inclusions are isotropically distributed, the distribution of $y$ is uniform in $\left[-\frac{L_{\rho}}{2}, \frac{L_{\rho}}{2}\right]$. Its probability density function $p_{y}$ then reads:

$$
p_{y}(y)=\frac{1}{L_{\rho}}
$$

There are three possibilities for the interaction between the crack and the FEV: 
1. the crack does not meet the inclusion and propagates in the matrix;

2. the crack meets the inclusion and by-passes it;

3. the crack meets the inclusion and crosses it.

Lebihain et al. (2020) described the conditions under which the by-pass mechanism prevail over the crossing one through a two-dimensional model based on Amestoy-Leblond's formulæ (Amestoy and Leblond, 1992; Leblond, 1999). It has been shown to reproduce accurately the crossing to by-pass transition on three-dimensional simulations where a crack interacts with spherical inclusions. The transition between both regimes is given by the following equation:

$$
\frac{G_{\mathrm{c}}^{\mathrm{inc}}}{G_{\mathrm{c}}^{\text {int }}}=\frac{1}{F_{\mathrm{I}, \mathrm{I}}(\theta)^{2}+F_{\mathrm{II}, \mathrm{I}}(\theta)^{2}}, \text { where } \theta=\arcsin \left(\frac{y}{d_{\mathrm{inc}}}\right)
$$

For a given toughness ratio $G_{\mathrm{c}}^{\text {inc }} / G_{\mathrm{c}}^{\text {int }}$, the inclusion is crossed for landing heights $y$ close to the equatorial plane of the spherical inclusion, where the by-pass angle $\theta_{\tan }$ is larger. It is however by-passed when the crack lands near the top where the tangent angle is low, making the by-pass easier. If the crack does not encounter the inclusion for $|y|>d_{\text {inc }} / 2$, it propagates in the matrix. This behavior can be summarized within a transition diagram plotted in Fig. 10.(b).
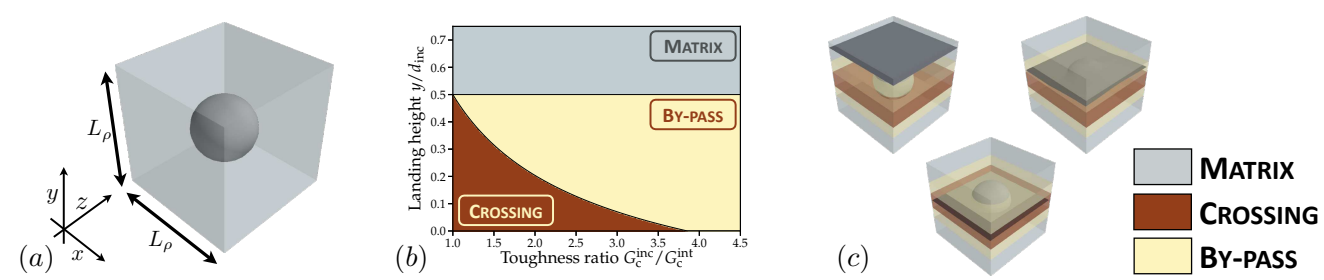

Figure 10: (a) Elementary volume (EV) of size $L_{\rho}$ constituted by a single spherical inclusion embedded within a cubical matrix of size $L_{\rho}$. (b) A crack landing at a height $y$ on the inclusion of diameter $d_{\text {inc }}$, toughness $G_{\mathrm{c}}^{\text {inc }}$ and interface toughness $G_{\mathrm{c}}^{\text {int }}$ can interact through one of three mechanisms (matrix cracking, inclusion crossing, or by-pass), which is inferred from Eq. (14). This interaction gives birth to a fractured elementary volume (FEV).

The microstructural properties of the inclusion and the landing height being independent variables, the probability density function reads:

$$
p_{\text {FEV }}\left(\omega_{\mathrm{F}}\right)=p_{y}(y) \cdot p_{d}\left(d_{\mathrm{inc}}\right) \cdot p_{\mathrm{inc}}\left(G_{\mathrm{c}}^{\mathrm{inc}}\right) \cdot p_{\mathrm{int}}\left(G_{\mathrm{c}}^{\mathrm{int}}\right)
$$

We note $\Omega_{\mathrm{F}}$ the statistical ensemble of possible realizations of FEV. The assemblage of all the FEV in $\Omega_{\mathrm{F}}$ is representative of the interaction between a crack and a disordered distribution of tough inclusions in the non-collective limit, when the mechanism selection in one FEV is independent of that in a neighboring FEV. One thus expects the proposed homogenization model to work well for small toughness contrast, for which the probability of by-pass is small. Indeed, we will see later that the out-of-plane front perturbations resulting from by-pass in a FEV can affect the mechanism selection in neighboring FEVs.

\subsection{The influence of the mechanisms of interaction: the equivalent coplanar element}

We now have to translate the three-dimensional FEV problem into a coplanar one. $G_{\mathrm{c}}^{\mathrm{cop}}(z, x)$ denotes the toughness field that the crack front actually visits during crack propagation in a given 
$\omega_{\mathrm{F}}=\left(y, d_{\text {inc }}, G_{\mathrm{c}}^{\text {inc }}, G_{\mathrm{c}}^{\text {int }}\right) \in \Omega_{\mathrm{F}}$. Matrix cracking and inclusion crossing corresponding to coplanar propagation, computing $G_{\mathrm{c}}^{\mathrm{cop}}$ is rather straightforward in that case.

For matrix cracking, it is given by:

$$
G_{\mathrm{c}}^{\mathrm{cop}}(z, x ; \omega)=G_{\mathrm{c}}^{\mathrm{mat}}
$$

For inclusion crossing, $G_{\mathrm{c}}^{\mathrm{cop}}$ reads:

$$
\left\{\begin{array}{l}
G_{\mathrm{c}}^{\mathrm{cop}}(z, x ; \omega)=G_{\mathrm{c}}^{\text {mat }}, \text { if } z^{2}+x^{2}>\left(\frac{d_{\text {inc }}}{2}\right)^{2}-y^{2} \\
G_{\mathrm{c}}^{\mathrm{cop}}(z, x ; \omega)=G_{\mathrm{c}}^{\text {inc }} \text { otherwise }
\end{array}\right.
$$

We now have to translate the impact of inclusion by-pass into an equivalent coplanar defect. Analytical results cannot be derived given the complexity of the non-linear equation of motion of Eq. (9). Yet it is possible to compute it numerically from efficient simulations on periodic arrangements of spherical inclusions.

In-plane distortions of the crack front and its dynamics are the local mirror images of the toughness field visited by the crack during propagation (Chopin et al., 2011; Patinet et al., 2013a). The equivalent coplanar toughness field $G_{\mathrm{c}}^{\mathrm{cop}}(z, x)$ can thus be inferred from the in-plane deformation of the crack front observed in periodic simulations of inclusion by-pass (see Fig. 11.(a-b)). We define then $G^{\text {cop }}$ as the distribution of ERR along the distorted crack front if its propagation was coplanar. Combining Eq. (3) with Eq. (5) in the limit where $f_{y}=0$ and $\theta=0, G^{\text {cop }}$ reads at first-order in the perturbation :

$G^{\mathrm{cop}}(z, t)=G^{\infty}(t)\left(1+2 \frac{\delta K_{\mathrm{I}}(z, t)}{K_{\mathrm{I}}^{\infty}(t)}\right)=G^{\infty}(t)\left(1-\frac{1}{\mathcal{L}} f_{x}(z, t)-\frac{1}{\pi} \mathrm{PV} \int_{-\infty}^{+\infty} \frac{f_{x}(z, t)-f_{x}\left(z^{\prime}, t\right)}{\left(z-z^{\prime}\right)^{2}} d z^{\prime}\right)$

Like the field $G_{\mathrm{c}}^{\text {frac }}$ of dissipated energy (see Appendix B), $G^{\text {cop }}$ can be tracked during crack propagation (see Fig. 11) and stored in a grid, each value $G^{\mathrm{cop}}(z, x)$ corresponding to the average of the equivalent coplanar ERR $G^{\mathrm{cop}}$ in this cell.

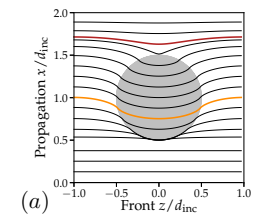

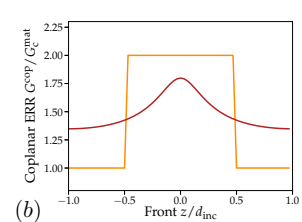

(b)
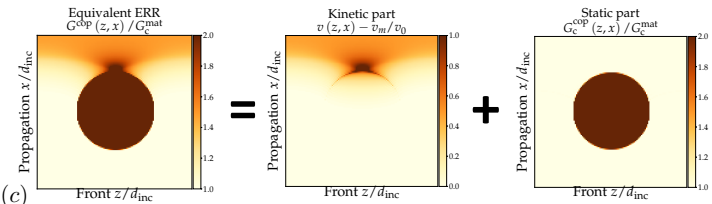

Figure 11: Construction of the equivalent coplanar element (ECE): (a) the in-plane deformations of the crack front during crack-inclusion interaction are linked to (b) an equivalent coplanar ERR $G^{\text {cop }}$ (associated to the crack front profile marked in red). Tracking the front distortions allows to construct (c) the field $G^{\text {cop }}$, which is then decomposed in a kinetic part that related to the depinning dynamics from the defect, and a static part that correspond to the equivalent coplanar element.

We first test this method on the in-plane crossing of an inclusion twice tougher than the matrix $G_{\mathrm{c}}^{\text {inc }}=2 G_{\mathrm{c}}^{\text {mat }}$ interacting with a crack landing at $y_{\text {landing }}=0$. The results are plotted in Fig. 11.(c). It turns out that such a definition of the equivalent coplanar toughness does not 
provide the expected toughness field of Eq. (17). Indeed, the proposed method does not take account of the rate-dependency $G^{\mathrm{cop}}(z, t)=G_{\mathrm{c}}[v(z, t)]$ of the fracture energy so that it is not able to distinguish the dissipation resulting from the raw toughness $G_{\mathrm{c}}(z, x)$ of the material from the one resulting from the micro-instabilities taking place during depinning from a defect (see (Chopin et al., 2018)). Thus $G^{\mathrm{cop}}$ corresponds to the kinetic equivalent coplanar toughness field.

In order to remove the impact of kinetic effects that are already embedded in Démery et al. (2014b)'s coplanar theory, we estimate the average crack velocity $v(z, x)$ on a grid and invert the kinetic law in Eq. (6). We have then access to the static equivalent coplanar toughness field $G_{\mathrm{c}}^{\text {cop }}$

$$
G_{\mathrm{c}}^{\mathrm{cop}}\left(z_{j}, x_{i}\right)=G^{\mathrm{cop}}\left(z_{j}, x_{i}\right)-G_{\mathrm{c}}^{\mathrm{mat}}\left(\frac{v\left(z_{j}, x_{i}\right)-v_{m}}{v_{0}}\right)
$$

Note that for a crack speed $\mathrm{v}$ equal to the driving speed $v_{m}$, both quantities are the same. As pictured in Fig. 11.(c), $G_{\mathrm{c}}^{\text {cop }}$ corresponds now to the expected equivalent coplanar toughness field for inclusion crossing. Besides, such a static field $G_{\mathrm{c}}^{\text {cop }}(z, x)$ happens to be independent of the inclusion spacing $L_{\rho}$, as verified from simulations on periodic arrangements of tough inclusions.

The equivalent defect is plotted in Fig. 12 for each of the three mechanisms considered in the present study. We notice that a slight change in the inclusion toughness modifies drastically the equivalent coplanar defect shape and its intensity, as the propagation mechanism shifts from in-plane to out-of-plane. In turn, it will also affect the effective toughness, as explained in the next part.
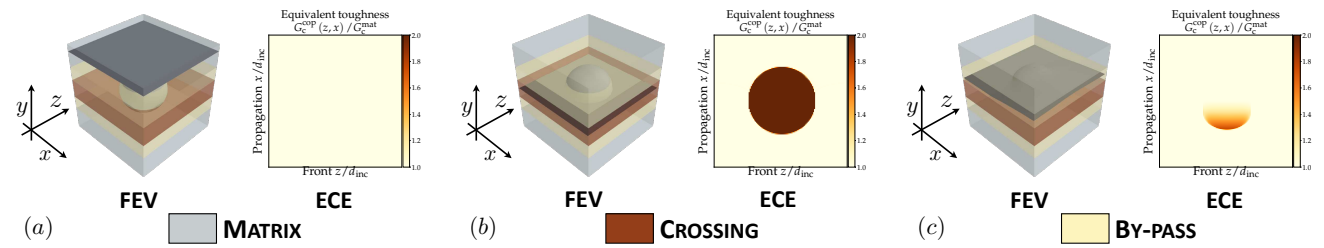

Figure 12: Fracture elementary volumes (FEVs) and equivalent coplanar elements (ECEs) associated with the three mechanisms considered in the study: (a) matrix cracking, (b) crossing and (c) by-pass at the transition

$G \underset{\mathrm{c}}{\mathrm{inc}}=3.854 G_{\mathrm{c}}^{\mathrm{mat}}$ from one mechanism to another for a crack landing on the equatorial plane $y=0$.

\subsection{Estimating the effective toughness of disordered composites: the reconstructed toughness distribution}

Thanks to the procedure described before combined with the interaction diagram of Fig. 10.(b), we can determine an equivalent coplanar element (ECE) for each FEV realization $\omega_{\mathrm{F}} \in \Omega_{\mathrm{F}}$. Under the ergodic assumption, the spatial average of an observable $f$ on $L_{z} \times L_{x}$, is equal to the ensemble average on both the surface of the ECEs $L_{\rho} \times L_{\rho}$ and the realizations $\Omega_{\mathrm{F}}$. This assumption writes as:

$$
\langle f(z, x)\rangle_{z \in\left[0, L_{z}\right], x \in\left[0, L_{x}\right]}=\left\langle f\left(z, x ; \omega_{\mathrm{F}}\right)\right\rangle_{z \in\left[0, L_{\rho}\right], x \in\left[0, L_{\rho}\right], \omega_{\mathrm{F}} \in \Omega_{\mathrm{F}}}
$$

Thus, the four parameters $\left\langle G_{\mathrm{c}}\right\rangle, \sigma, \xi_{z}$ and $\xi_{x}$ required to predict $G_{\mathrm{c}}^{\text {eff }}$ can be inferred from the assemblage of all the ECEs associated with each FEV realization $\omega_{\mathrm{F}} \in \Omega_{\mathrm{F}}$ from the following 
expressions:

$$
\left\{\begin{array}{l}
\left\langle G_{\mathrm{c}}\right\rangle=\frac{1}{L_{\rho}^{2}} \iiint_{\omega_{\mathrm{F}}, x, z} G_{\mathrm{c}}^{\mathrm{cop}}\left(z, x ; \omega_{\mathrm{F}}\right) p_{\mathrm{FEV}}\left(\omega_{\mathrm{F}}\right) d z d x d \omega_{\mathrm{F}} \\
\left\langle G_{\mathrm{c}}^{2}\right\rangle=\frac{1}{L_{\rho}^{2}} \iiint_{\omega_{\mathrm{F}}, x, z} G_{\mathrm{c}}^{\mathrm{cop}}\left(z, x ; \omega_{\mathrm{F}}\right)^{2} p_{\mathrm{FEV}}\left(\omega_{\mathrm{F}}\right) d z d x d \omega_{\mathrm{F}} \\
\xi_{z}=\frac{1}{L_{\rho}^{2}} \int_{\omega_{\mathrm{F}}} \xi_{z}\left(\omega_{\mathrm{F}}\right) p_{\mathrm{FEV}}\left(\omega_{\mathrm{F}}\right) d \omega_{\mathrm{F}} \text { and } \xi_{x}=\frac{1}{L_{\rho}^{2}} \int_{\omega_{\mathrm{F}}} \xi_{x}\left(\omega_{\mathrm{F}}\right) p_{\mathrm{FEV}}\left(\omega_{\mathrm{F}}\right) d \omega_{\mathrm{F}} \\
\sigma\left(G_{\mathrm{c}}\right)^{2}=\left\langle G_{\mathrm{c}}^{2}\right\rangle-\left\langle G_{\mathrm{c}}\right\rangle^{2}
\end{array}\right.
$$

where the symbol $\int_{\omega} \int_{x} \int_{z}$ denotes the integrals over $\omega_{\mathrm{F}} \in \Omega_{\mathrm{F}}, z \in\left[0, L_{\rho}\right]$ and $x \in\left[0, L_{\rho}\right]$.

Examples of the equivalent distribution of toughness $G_{c}^{\text {coplanar }}(z, x)$ resulting from the assemblage of all ECE realizations computed through the homogenization procedure are plotted in Fig. 13.
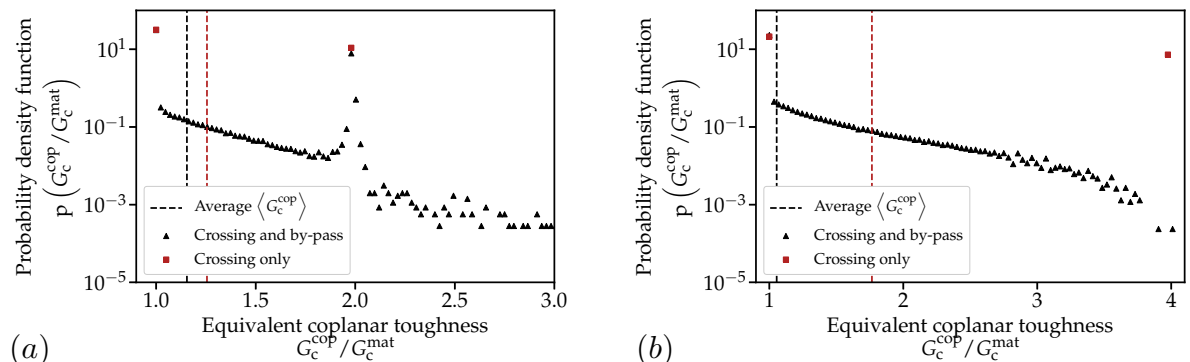

Figure 13: Equivalent coplanar toughness distribution accounting for the competing crossing and by-pass mechanisms : the toughness distribution is extracted from the procedure described in Section 4.4 for an inclusion density $\rho_{\text {inc }}=25 \%$ and inclusion toughness $G_{\mathrm{c}}^{\text {inc }}=2 G_{\mathrm{c}}^{\text {mat }}$ (a) and $G_{\mathrm{c}}^{\text {inc }}=4 G_{\mathrm{c}}^{\text {mat }}(\mathrm{b})$. Red square markers correspond to the case where the sole crossing mechanism is accounted for while black triangular markers combine the competing effect of both crossing and by-pass mechanisms.

By combining Eq. (10) with Eq. (21), it is possible to predict the effective toughness in the case of non-coplanar propagation. Section 4.6 is dedicated to the validation of the proposed approach through the influence of the inclusion toughness on the effective toughness studied in Section 3.3.

\subsection{Model predictions for increasing inclusion toughness}

We first use the homogenization framework to investigate the influence of the inclusion toughness on the effective toughness and compare the predictions to the numerical results plotted in Fig. 8.

In Section 3.3, we considered a composite with only one type of inclusions of size $d$ and toughnesses $G_{\mathrm{c}}^{\mathrm{inc}}$ and $G_{\mathrm{c}}^{\mathrm{int}}$. The probability density function $p_{\mathrm{FEV}}$ of a FEV realization $\omega_{\mathrm{F}} \in \Omega_{\mathrm{F}}$ then reads:

$$
p_{\mathrm{FEV}}\left(\omega_{\mathrm{F}}\right)=\frac{1}{L_{\rho}} \cdot \delta\left(d_{\mathrm{inc}}-d\right) \cdot \delta\left(G_{\mathrm{c}}^{\text {inclusion }}-G_{\mathrm{c}}^{\mathrm{inc}}\right) \cdot \delta\left(G_{\mathrm{c}}^{\text {interface }}-G_{\mathrm{c}}^{\mathrm{int}}\right)
$$


where $\delta$ is the Dirac function.

We then go through each step of the homogenization procedure described in Section 4.1 and compare the homogenization-based predictions to the simulations results in Fig. 14.

We observe that the homogenization framework allows not only to predict the overall fracture properties of composite materials but also to quantify the influence of microstructural parameters on the effective toughness. When the inclusions get tougher, by-pass interactions progressively prevail over inclusion crossing and two mechanisms are at play:

- First, the toughening contribution of by-passed inclusions does not increase with the inclusion toughness (Lebihain et al., 2020), while that of the crossed inclusion keeps increasing (Gao and Rice, 1989). Indeed, when the inclusion is by-passed, the crack propagates along the interface between the inclusion and the matrix so that the toughness of the inclusion is no more relevant in the process. The toughening contribution of by-passed inclusions is then frozen.

- Second, when the mechanism shifts from crossing to by-pass, the equivalent coplanar defect the crack actually experiences becomes weaker (see Fig. 12). By-passed inclusions then effectively weakens the material.
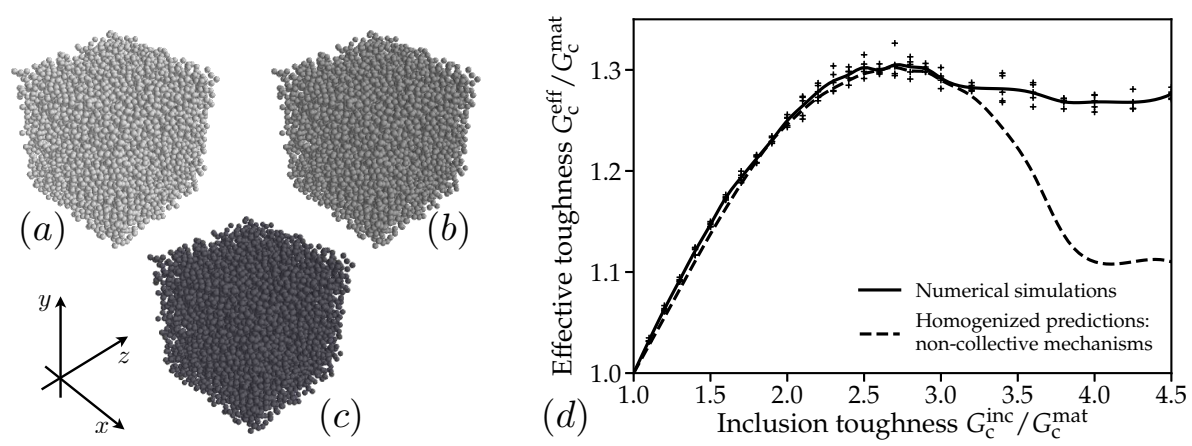

Figure 14: Impact of the inclusion toughness $G_{\mathrm{c}}^{\text {inc }}$ on the effective toughness $G_{\mathrm{c}}^{\text {eff }}$ for the coupling of crack trapping and crack deflection : the averaged effective toughness measured from numerical simulations following Eq. (7) (in solid black line) is compared to theoretical predictions of the homogenization framework (in dashed black line). Cross markers represent individual simulation points.

The first effect accounts for the progressive loss of toughening rate $\frac{\partial G_{c}^{\text {eff }}}{\partial G_{c}^{\text {inc }}}$. The second effect explains the decrease of the effective toughness with the toughness inclusion after the peak. Within our homogenization procedure, this effect results from the decrease of both the average value $\left\langle G_{\mathrm{c}}\right\rangle$ and the standard deviation $\sigma$ of the equivalent coplanar distribution (see Fig. 13). Combining both effects, this explains why the effective toughness reaches a maximum for $G_{\mathrm{c}}^{\text {inc }} \simeq$ $2.7 G_{\mathrm{c}}^{\mathrm{mat}}$ and then decreases for larger inclusion toughness levels. This decrease in overall toughening of the composite could be promoted in presence of weak interfaces $\left(G_{\mathrm{c}}^{\text {inc }}<G_{\mathrm{c}}^{\text {mat }}\right)$ that promote inclusion by-pass. These results are not shown in the present paper for clarity and brevity reasons, but can be found in Lebihain (2019). 
We notice that the homogenization-based predictions are in excellent agreement with numerical results up to $G_{\mathrm{c}}^{\mathrm{inc}} \simeq 3 G_{\mathrm{c}}^{\mathrm{mat}}$. This shows that the proposed framework contains the fundamental ingredients for the homogenization of fracture properties in disordered media. The homogenization framework reproduces with great accuracy the loss of toughening rate as well as the position and the value of the maximum effective toughness. Above this inclusion toughness level, the proposed model predicts a subsequent decrease of the effective toughness but over-estimates it substantially.

Such a discrepancy can have multiple origins, which can be related to each step of the homogenization procedure: the non-collective hypothesis for the FEV's mechanism selection, the determination of the equivalent coplanar defect, or the homogenization framework for coplanar propagation. In particular, the non-collective hypothesis might be questionable for large inclusion toughness ratios $G_{\mathrm{c}}^{\mathrm{inc}} / G_{\mathrm{c}}^{\text {mat }}$ since more frequent by-pass events occur. It may challenge the assumption that the crack is perfectly plane when it lands on an inclusion and thus modify the way crack and inclusions interact with each other. In Fig. 15, we plot the respective probabilities of the occurrence of the crossing and by-pass mechanisms during the computed interaction of a crack with a large distribution of inclusions. We see that theoretical predictions from Eq. (14) are only valid at a low toughness ratio $G_{\mathrm{c}}^{\text {inc }} / G_{\mathrm{c}}^{\text {mat }}=2$ (Fig. 15.(a)) while they differ from numerically observed interactions at high toughness ratio $G_{\mathrm{c}}^{\text {inc }} / G_{\mathrm{c}}^{\text {mat }}=4$ (Fig. 15.(b)). In particular, a larger portion of inclusion is crossed, which accounts for the mismatch in the plateau value of the effective toughness curve in Fig. 14.
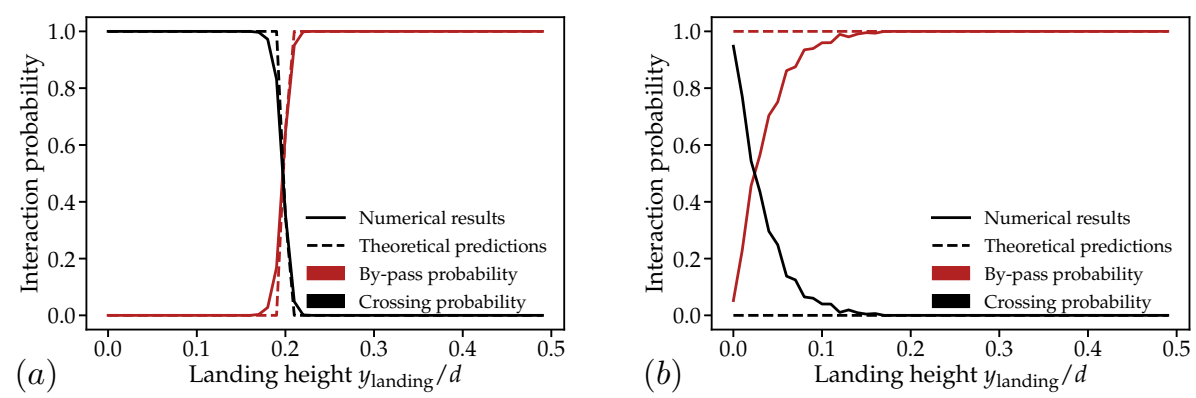

Figure 15: Probability of the crossing and by-pass mechanisms as a function of the crack landing position $y_{\text {landing }}$ : the probability is calculated via numerical simulation of a crack interacting with an inclusion distribution of density $\rho_{\text {inc }}=25 \%$ and toughness $G_{\mathrm{c}}^{\text {inc }}=2 G_{\mathrm{c}}^{\text {mat }}$ (a) and $G_{\mathrm{c}}^{\text {inc }}=4 G_{\mathrm{c}}^{\text {mat }}($ b). Numerical results in solid lines are compared with theoretical predictions from Eq. (14) in dashed lines.

This discrepancy results from the landing on an inclusion with a non-zero angle, as a result of its interaction with another inclusion met previously. This illustrates the need for future extensions of the proposed model to take into account possible interferences between neighboring inclusions. Such an extension has been proposed in Lebihain (2019) and has been shown to significantly improve model predictions. 


\section{Towards tailored composite with increased fracture toughness}

The objective of this last section is twofold: (i) to investigate the impact of microstructural properties on the effective toughness of disordered materials; (ii) to validate our homogenization model by comparing numerical results to analytical homogenization-based predictions. The homogenization model proposed in Section 4 provides physical insights on the effect of microstructural parameters on $G_{\mathrm{c}}^{\text {eff }}$. It consequently provides new design strategies to improve the material toughness by varying either the material disorder (and consequently $\left\langle G_{\mathrm{c}}\right\rangle, \sigma, \xi_{z}, \xi_{x}$ ) or the interaction mechanisms (and consequently the equivalent coplanar element of Section 4.4). These two different strategies are illustrated by investigating respectively the influences of the inclusion density in Section 5.1 and the inclusion shape in Section 5.2.

\subsection{Toughening from material disorder: the influence of the inclusion density}

We first explore how to toughen a brittle material by tuning the microstructural disorder. As a result, we focus first on the influence of the inclusion density on the effective toughness of heterogeneous materials. We consider crack propagation in large monodisperse distributions of spherical inclusions of diameter $d$ at varying density levels $\rho_{i n c} \in\{10 \%, 50 \%\}$. The inclusion distributions are generated using the procedures detailed in Appendix A. As before, the inclusion toughness varies from $G_{\mathrm{c}}^{\mathrm{inc}}=G_{\mathrm{c}}^{\text {mat }}$ to $G_{\mathrm{c}}^{\mathrm{inc}}=4.5 G_{\mathrm{c}}^{\text {mat }}$, while the interface shares the fracture properties of the matrix $G_{\mathrm{c}}^{\text {int }}=G_{\mathrm{c}}^{\text {mat }}$. Some examples of the considered distributions are given in Fig. 16.(a-c).
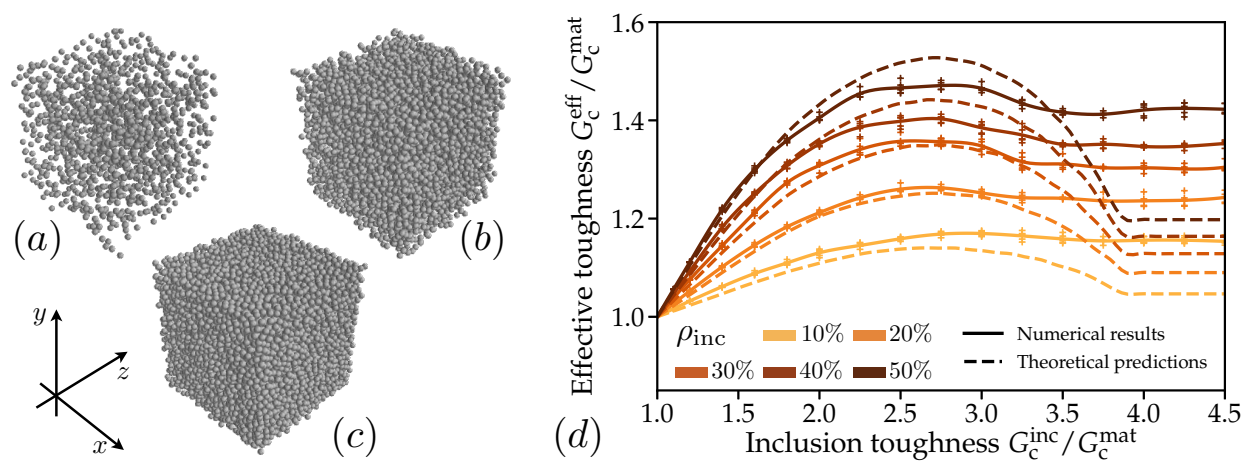

Figure 16: Impact of the inclusion density $\rho_{\text {inc }}$ on the effective toughness $G_{\mathrm{c}}^{\mathrm{eff}}:(\mathrm{a}-\mathrm{c})$ the crack interacts with large distributions of spherical inclusions with varying toughness and density. (d) The effective toughness is estimated from the crack evolution following Eq. (7). Numerical results (in solid lines) are compared to theoretical predictions of the homogenization model (in dashed lines).

As could be anticipated, a denser distribution of inclusions induces an additional toughening of the composite material through the increase of the average $\left\langle G_{\mathrm{c}}\right\rangle$ of the visited toughness distribution and its standard variation $\sigma$. Interestingly, the position of the maximum of $G_{\mathrm{c}}^{\text {eff }}$ at $G_{\mathrm{c}}^{\text {inc }} \simeq 2.7 G_{\mathrm{c}}^{\text {mat }}$ does not vary with the inclusion density. Indeed, the position of the maximum results from the crossing to by-pass transition, which is not affected by the inclusion density (Lebihain et al., 2020). We see that the homogenization framework gives quantitative predictions up to $G_{\mathrm{c}}^{\text {inc }} \simeq 3 G_{\mathrm{c}}^{\text {mat }}$, which accounts for a precise determination of the maximum position. 
We can nonetheless notice some mismatch for both high inclusion densities, where strong outof-plane perturbations challenge our hypothesis of zero landing angle, and for low inclusion densities, due to finite-size effects ${ }^{7}$. Above $3 G_{\mathrm{c}}^{\text {mat }}$, the model predictions are no more quantitative due to collective effects on the interaction mechanism selection as explained before.

We now seek to determine an analytical expression linking the inclusion density and the effective toughness. For each realization $\omega_{\mathrm{F}} \in \Omega_{\mathrm{F}}$ of a FEV, we first define an equivalent coplanar defect of area $S_{\text {defect }}$ and toughness contrast $c_{\text {defect }}$ so that the toughness field of the ECE $G_{\mathrm{c}}^{\text {cop }}$ reads:

$$
\left\{\begin{array}{l}
G_{\mathrm{c}}^{\mathrm{cop}}(z, x)=G_{\mathrm{c}}^{\mathrm{mat}}\left[1+c_{\text {defect }}(z, x)\right] \text { inside the defect } \\
G_{\mathrm{c}}^{\mathrm{cop}}(z, x)=G_{\mathrm{c}}^{\text {mat }} \text { outside of it }
\end{array}\right.
$$

Examples of such a coplanar toughness field and contrast defect $c_{\text {defect }}$ are given in Fig. 12.
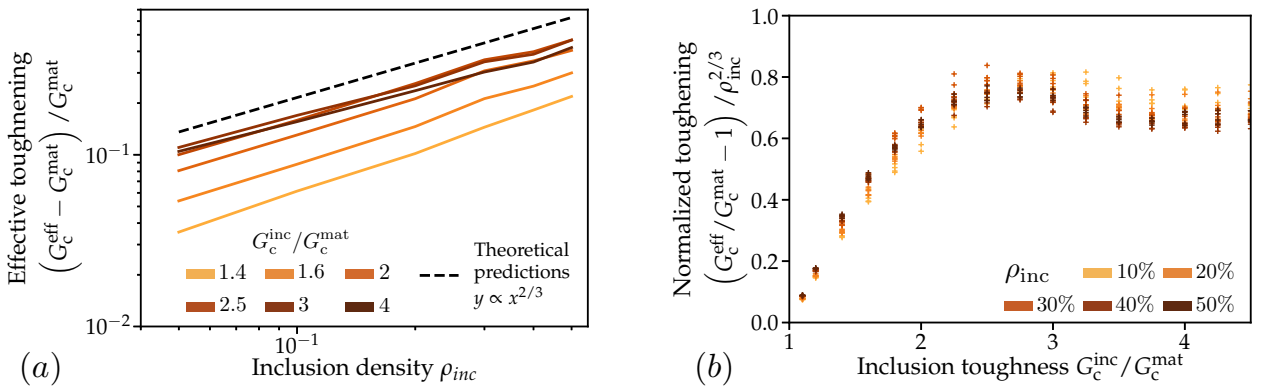

Figure 17: Impact of the inclusion density on the effective toughness: (a) scaling between $\left(G_{\mathrm{c}}^{\text {eff }}-G^{\text {mat }}\right) / G_{\mathrm{c}}^{\text {mat }}$ and the inclusion density $\rho_{\text {inc }}$ and comparison with the theoretical prediction of Eq. (25); (b) rescaling of the curves of Fig. 17.(a) using the relation (25)

We then use the homogenization procedure of Eq. (21) to compute the parameters:

$$
\left\{\begin{array}{l}
\left\langle G_{\mathrm{c}}\right\rangle / G_{\mathrm{c}}^{\mathrm{mat}}=1+\frac{1}{L_{\rho}^{2}}\left\langle c_{\text {defect }} S_{\text {defect }}\right\rangle \\
\sigma^{2}=\frac{1}{L_{\rho}^{2}}\left(\left\langle c_{\text {defect }}^{2} S_{\text {defect }}\right\rangle-2\left\langle c_{\text {defect }} S_{\text {defect }}\right\rangle\right)+\frac{1}{L_{\rho}^{4}}\left\langle c_{\text {defect }} S_{\text {defect }}\right\rangle^{2}
\end{array}\right.
$$

where $S_{\text {defect }}$ and $c_{\text {defect }}$ are the area and the toughness contrast characterizing the equivalent planar toughness field. $\langle\cdot\rangle$ denotes the average on both the surface $L_{\rho} \times L_{\rho}$ of the ECEs and the realizations $\Omega_{\mathrm{F}}$.

Using these expressions, Eq. (10) provides at the lowest order in $\rho_{\text {inc }}$ :

$$
\frac{G_{\mathrm{c}}^{\mathrm{eff}}-G_{\mathrm{c}}^{\mathrm{mat}}}{G_{\mathrm{c}}^{\mathrm{mat}}} \propto \rho_{\mathrm{inc}}^{2 / 3}
$$

\footnotetext{
${ }^{7}$ The crack is then only pinned by rare defects and propagates in the weak pinning regime. The associated Larkin length is then larger than the system size, $L_{c} \leq L_{z}$, a regime that is included in our theoretical model.
} 
This scaling, which corresponds to the projection of a volumetric density onto an area density, is compared with numerical simulations in Fig. 17.(a). The rescaling based on Eq. (25) is shown in Fig. 17.(b).

\subsection{Toughening by tuning the interaction mechanisms: the impact of the inclusion shape}

We now want to improve the material toughness by tuning the mechanisms selected by the crack during its interaction with tough inclusions. Lebihain et al. (2020) showed that the mechanism selection and the subsequent toughening contribution were largely governed by the inclusion geometry. We show here that changing the inclusion shape from sphere to a cube can strongly increase the inclusion-induced reinforcement, a feature that is grasped quantitatively by the homogenization framework proposed in Section 4.

We consider monodisperse distributions of cubical inclusions at a density $\rho_{\text {inc }}=20 \%$, as depicted in Fig.18.(a). Each cubical inclusion is described by its edge length $d$ and its inclination $\beta_{\text {inc }}$, defining its rotation in a clockwise direction around the $z$-axis (see Fig. 19.(a-b)). The inclusion toughness varies from $G_{\mathrm{c}}^{\text {inc }}=G_{\mathrm{c}}^{\text {mat }}$ to $G_{\mathrm{c}}^{\text {inc }}=4.5 G_{\mathrm{c}}^{\text {mat }}$, while the interfaces share the same fracture properties as the matrix. The results of 90 simulations are plotted in Fig.18.(d) and compared to three-dimensional crack propagation in disordered materials with spherical inclusions (Fig.18.(b)) as well as coplanar crack propagation in disordered fiber composites (Fig.18.(c)).
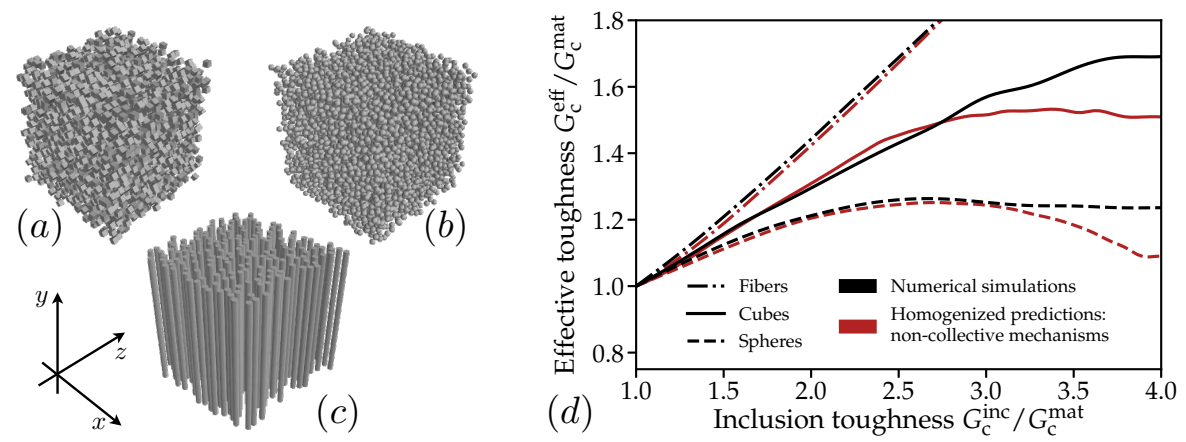

Figure 18: Impact of the inclusion shape on the effective toughness $G_{\mathrm{c}}^{\text {eff }}:$ the crack interacts with randomly distributed of (a) cubical inclusions, (b) spherical inclusions, or (c) fibers with varying toughness but constant density $\rho_{\text {inc }}=20 \%$. (d) The effective toughness is estimated from the crack evolution following Eq. (7). Numerical results (in black lines) are compared to theoretical predictions of the homogenization model (in red lines).

First, we observe that the reinforcement induced by cubical inclusions is three times larger than the one triggered by spherical inclusions. Such a shift in behavior emphasizes the strong dependence of the macroscopic fracture properties on the material features at a microstructural scale. Second, we notice that, up to $G_{\mathrm{c}}^{\text {inc }} / G_{\mathrm{c}}^{\text {mat }} \simeq 2.5$, the effective toughness emerging from microstructures with cubical inclusions remains close to the effective toughness of fiber-reinforced composites, for which crack propagation is planar. This suggests that the by-pass of inclusions that controls the weakening observed at large inclusion toughness, is much rarer for cubic inclusions than for spherical ones. 
The cubical geometry of the inclusions presents indeed an interesting property with respect to the spherical inclusion: the deflection angle $\theta_{\tan }$ remains constant during by-pass. This feature prevents the crack from realigning with the direction $(O x)$ imposed by the macroscopic Mode I loading. As the crack goes out-of-plane and by-passes the inclusion, it is dragged back to the main fracture plane by an increasing long-range Mode II contribution (see Eq.3), leading it to leave the matrix/inclusion interface and ultimately cross the inclusion. The crack-inclusion interaction then involves not two, but three mechanisms: a crossing one where the crack penetrates the inclusion, a by-pass one where it propagates along the interface, and a repenetration one that constitutes a mix between the two previous ones during which the crack crosses the inclusion after an initial by-passing phase. Mechanism selection is controlled by the height $y_{\text {landing }}$ at which the cracks lands on the inclusion, its inclination $\beta_{\text {inc }}$, which controls the deflection angle $\theta_{\tan }$, as well as its fracture properties $G_{\mathrm{c}}^{\text {inc }}$. It can be summarized in the transition diagram of Fig. 19, which has been computed from 22,000 periodic simulations.
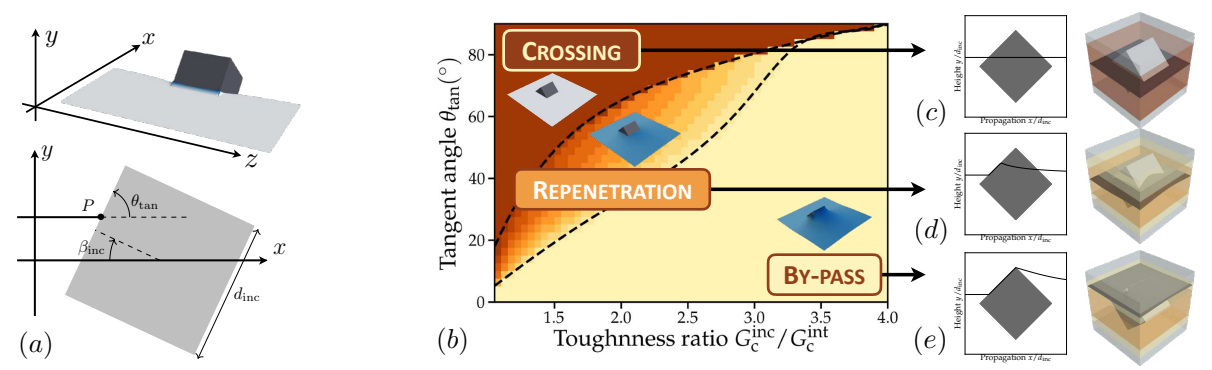

Figure 19: Crack interacting with a cubical inclusion of edge length $d$ and inclination $\beta_{\text {inc: }}$ (a) three-dimensional view and projection in the $(x M y)$ plane. (b) Transition diagram obtained from 22,000 simulations with various toughness ratios $G_{\mathrm{c}}^{\text {inc }} / G_{\mathrm{c}}^{\text {mat }}$, inclusion inclinations $\beta_{\text {inc }}$, and landing heights $y_{\text {landing }} / d$. Three interaction mechanisms are observed: the (c) crossing and (d) by-pass mechanisms are separated by a transient phase of (c) repenetration, where the crack starts to by-pass the inclusion before crossing it.

We then go through the homogenization procedure developed in Section 4 that is adopted here to predict the reinforcement of a material by disordered distributions of cubical inclusions. For each FEV $\left(y_{\text {landing }}, d_{\text {inc }}, \beta_{\text {inc }}, G_{\mathrm{c}}^{\text {inc }}, G_{\mathrm{c}}^{\text {int }}, y\right) \in \Omega_{\mathrm{F}}$ (see Fig. 20.(a-c)), one can infer the interaction mechanism selected by the crack from the transition diagram of Fig. 19.(b) and the associated ECE (see Fig. 20.(a-c)) computed from thousands of efficient periodic simulations. The assemblage of the ECEs for all possible realizations finally allows for the determination of the average $\left\langle G_{\mathrm{c}}\right\rangle$, standard deviation $\sigma\left(G_{\mathrm{c}}\right)$, and correlation lengths $\xi_{z}$ and $\xi_{x}$ of the equivalent coplanar distribution under the ergodic assumption. Equation (10) finally leads to the prediction of the effective toughness. Such predictions are plotted in red lines in Fig. 18. We observe that the proposed model reproduces the additional toughening triggered by the change in the geometry up to $G_{\mathrm{c}}^{\text {inc }} \simeq 3 G_{\mathrm{c}}^{\text {mat }}$. After that, the selected mechanisms start to depend on interactions with neighboring particles.

The homogenization framework provides rich insights into the physical mechanisms lying behind the predicted toughening. First, we observe on Fig. 19.(b) that the crossing to by-pass transition is no more sharp, as was the case for spherical inclusions, but involves a transient repenetration phase, where the crack explores both the toughness of the interface during the 
initial by-pass and that of the inclusion during its subsequent crossing. The repenetration mechanism then generates a contribution to the ultimate material toughening that is similar to inclusion crossing, as it can be seen from the respective ECEs plotted in Fig. 20.(a-c). It extends the regime over which the composite is reinforced by crack trapping, accounting for the increased toughening observed up to $G_{\mathrm{c}}^{\text {inc }} \simeq 3 G_{\mathrm{c}}^{\text {mat }}$. Second, the cubical geometry improves the toughening potential of crack deflection by preventing the crack to realign with the direction of propagation $(O x)$, as can be observed by comparing ECEs for the by-pass of a spherical inclusion (Fig. 12.(b)) and a cubical one (Fig. 20.(c)). This effect accounts for the higher effective toughness plateau which appears from $G_{\mathrm{c}}^{\text {inc }} \simeq 3.7 G_{\mathrm{c}}^{\text {mat }}$ as well as the absence of a local maximum on $G_{\mathrm{c}}^{\text {eff }}$.
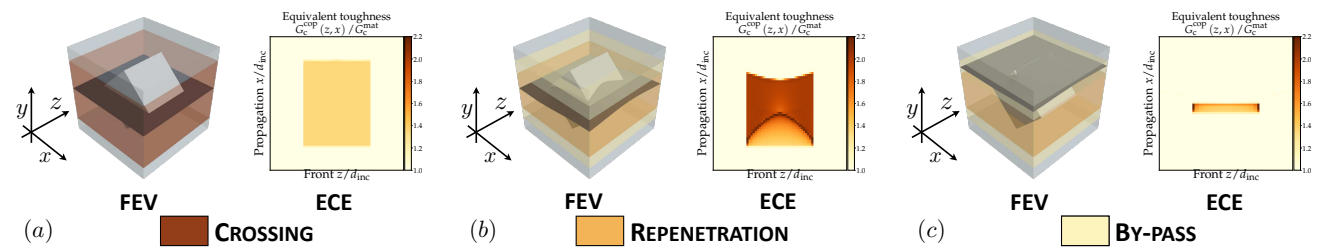

Figure 20: Homogenization scheme for the prediction of effective fracture properties of a composite material with cubical inclusions: fractured elementary volumes (FEVs) and associated equivalent coplanar elements (ECEs) are inferred for each three mechanisms of interaction - (a) crossing, (b) repenetration, and (c) by-pass.

Thus, a slight change in the inclusion geometry can have dramatic consequences on the effective fracture properties by modifying the mechanisms of interaction occurring at the crack tip as well as their individual toughening potential. Their ultimate contribution to the overall material toughening can be grasped in a disordered setting through the homogenization framework of Section 4.

\subsection{Extension of the proposed approach to a broader class of material microstructures}

The LEFM-based numerical method proposed in Section 2 allows to model crack interaction with large disordered distributions of tough inclusions through both crossing and by-pass mechanisms. Its unprecedented numerical performances represent a prerequisite to address the question of the homogenization of brittle fracture properties from a numerical point of view. Such performances are dependent on strong assumptions that limits its domain of applicability, namely the inclusions and the matrix are assumed to share the same elastic properties, and inclusion debonding or micro-cracking processes ahead of the crack tip are absent. However, the numerical simulations of Section 2 should be considered as a case study to challenge and validate the homogenization framework proposed in Section 4 that aims at a much wider scope. Indeed, it provides the core ingredients to predict the effective toughness of disordered materials for a broader class of heterogeneities and interaction mechanisms, as is detailed below.

First, the perturbative approach of Section 2 is restricted to spatial variations of toughness only. To overcome this limitation, the interaction between a crack and a single inclusion could be addressed with more general computational tools (e.g. phase-field models (Clayton and Knap, 2014; Nguyen, 2015; Nguyen et al., 2017a)) to identify the mechanisms involved during crack 
propagation and the conditions under which one mechanism prevail over the others ${ }^{8}$. From these simulations, one could follow the very same approach as proposed in Section 4.3 and construct a catalog of fractured elementary volumes $\omega_{\mathrm{F}}$ to each of which would be ascribed a given mechanism and an associated probability $p_{\mathrm{FEV}}\left(\omega_{\mathrm{F}}\right)$. Second, the individual contribution of each FEV to material toughening could be estimated by looking at the in-plane deformation of the crack front during the crack-inclusion interaction, following the approach developed in Section 4.4. Third, one could reconstruct the toughness field that the crack actually experiences during crack propagation from the assemblage of all FEVs under the ergodic assumption, and predict the effective toughness of disordered materials using tools borrowed from statistical physics like in Section 4.5.

This approach could be used to explore the impact of microstructural features (e.g. fracture properties and geometry of the inclusions) on the effective fracture properties and design new strategies to improve the overall toughness of composite materials by tuning either the material disorder, or directly the mechanisms occurring at the crack-tip during the crack-inclusion interaction.

\section{Conclusion}

This study aimed at building a comprehensive homogenization framework that predicts intrinsic effective fracture properties, taking into account the decisive impact of both the material disorder and the crack-tip mechanisms of interaction of a crack with microstructural heterogeneities. Our approach builds on the specificities of brittle fracture : (i) an evolution problem that (ii) is related to a structural problem as required by Griffith's theory and (iii) involves dissipative processes localized at the crack tip.

First, one has to model crack propagation in heterogeneous materials to predict effective fracture properties. Use was made for this purpose of the semi-analytical method developed in (Lebihain et al., 2020), whose core ingredients were recalled in Section 2. Based on the perturbative approach of Linear Elastic Fracture Mechanics (Gao and Rice, 1986; Movchan et al., 1998), it allows to efficiently compute the Stress Intensity Factors during the interaction of a crack with spatial heterogeneities of material toughness. The combination of the discontinuity of toughness at the inclusion/matrix interface and the Generalized Maximum Energy Release Rate criterion (GMERR) (He and Hutchinson, 1989; Gurtin and Podio-Guidugli, 1998) allows to model the competition between the in-plane crossing of a tough inclusion and its out-of-plane by-pass. The three-dimensional crack propagation in large disordered distributions of tough inclusions can be computed with remarkable computational efficiency, a feature that provides the basic tools to address the question of the homogenization of brittle fracture properties.

Second, intrinsic effective fracture properties can be defined by decoupling the material problem from the structural one through the choice of suitable boundary conditions. In Section 3, we revisited the concept of effective toughness $G_{\mathrm{c}}^{\text {eff }}$, and unified the three definitions proposed so far, namely :

${ }^{8}$ Three-dimensional simulations of a crack interacting with a single inclusion have already been computed with phasefield models at acceptable computational times (Nguyen et al., 2017b). 
- the effective toughness as the averaged fracture energy dissipated by the fracture process $\left\langle G_{\mathrm{c}}^{\mathrm{frac}}\right\rangle$;

- the average value $G_{\text {mean }}^{\infty}$ of the ERR during crack propagation, taking advantage of the coupling between the material problem and the structural one;

- the maximum value $G_{\max }^{\infty}$ of the ERR during crack propagation.

In particular, we showed that these three definitions converge to a single value under the scaleseparation condition :

$$
\mathcal{L}=-G^{\infty} / \frac{\partial G^{\infty}}{\partial x} \gg d
$$

where the structural length $\mathcal{L}$ is related to the boundary conditions and the specimen geometry and $d$ is the heterogeneity size. This condition enforces further restrictions on the experimental set-ups designed to measure effective fracture properties, as well as on the boundary conditions used in numerical simulations of crack propagation in composite materials.

Third, the influence of the material disorder and the interaction mechanisms on the effective toughness can be grasped within a comprehensive homogenization framework. Inspired by micromechanics and statistical physics, it relies on an accurate description of the conditions (e.g. mechanical and morphological properties of the inclusions) under which an interaction mechanism prevails over the others and its individual contribution to material toughening. These contributions are then added up thanks to the framework developed by Démery et al. (2014b) that accounts for the toughening induced by the collective pinning of a planar crack by an assembly of randomly distributed obstacles. This approach has been validated through systematic comparisons between homogenization-based predictions and numerical results produced by the perturbative model. It successfully predicts the impact of the competing crossing and by-pass mechanisms triggered by toughness heterogeneities on the effective toughness of heterogeneous brittle materials. It has been shown to yield quantitative predictions for spherical and cubical inclusions up to 3 times tougher than the matrix $G_{\mathrm{c}}^{\text {inc }}=3 G_{\mathrm{c}}^{\text {mat }}$, at densities up to $\rho_{\text {inc }}=50 \%$ in a non-collective situation, where the mechanism selection is not influenced by previous interactions. A collective scheme has been proposed in Lebihain (2019) to extend the predictive range of the proposed homogenization framework.

Both numerical and theoretical results highlight the substantial impact of a wide range of microstructural parameters on material reinforcement, from which one can infer guidelines for microstructural design :

- no matter the toughening mechanism involved, the toughness increase has been shown to scale with the inclusion density as $\rho_{\text {inc }}^{2 / 3}$;

- the by-pass mechanism is detrimental to material reinforcement, since it activates crack deflection whose contribution to material toughening is often lesser than that of crack trapping ;

- one can vary the inclusion geometry to delay inclusion by-pass by promoting inclusion repenetration. One can use cubical inclusions or alternatively pellets to promote such a mechanism. 
Finally, if the proposed homogenization method has been applied here to inclusion crossing and inclusion by-pass, it aims at predicting effective brittle fracture properties in a much wider scope. Much richer crack-inclusion mechanisms of interaction (e.g. inclusion debonding or crack denucleation/renucleation at the interface of an elastic heterogeneity) can be addressed through powerful computational methods (e.g. phase-field models (Hossain et al., 2014; Clayton and Knap, 2014; Nguyen, 2015; Nguyen et al., 2017a; Brach et al., 2019) of crack propagation in heterogeneous materials). The individual contribution of the competing toughening mechanisms can be inferred by looking at the resulting in-plane deformations of the crack front that mirrors the toughness the crack actually experiences during its propagation. These contributions are then used to predict the effective toughness of disordered materials, following the method proposed in Section 4. Such an approach paves the way for the rational design of composites with unprecedented failure properties.

\section{Acknowledgments}

The authors gratefully thank Damien Vandembroucq, Vincent Deméry and Alberto Rosso for fruitful discussions.

\section{References}

Alava, M.J., Nukala, P.K.V.V., Zapperi, S., 2006. Statistical models of fracture. Advances in Physics 55, 349-476.

Amestoy, M., Leblond, J.B., 1992. Crack paths in plane situations II. detailed form of the expansion of the stress intensity factors. International Journal of Solids and Structures 29, 465-501.

Barés, J., Hattali, M., Dalmas, D., Bonamy, D., 2014. Fluctuations of global energy release and crackling in nominally brittle heterogeneous fracture. Physical Review Letters 113, 264301.

Bonamy, D., 2009. Intermittency and roughening in the failure of brittle heterogeneous materials. Journal of Physics D: Applied Physics 42, 214014.

Bonamy, D., Bouchaud, E., 2011. Failure of heterogeneous materials: A dynamic phase transition? Physics Reports 498, 1-44.

Bower, A., Ortiz, M., 1991. A three-dimensional analysis of crack trapping and bridging by tough particles. Journal of the Mechanics and Physics of Solids 39, 815-858.

Bower, A.F., Ortiz, M., 1990. Solution of three-dimensional crack problems by a finite perturbation method. Journal of the Mechanics and Physics of Solids 38, 443-480.

Brach, S., Hossain, M.Z., Bourdin, B., Bhattacharya, K., 2019. Anisotropy of the effective toughness of layered media. Journal of the Mechanics and Physics of Solids 131, 96-111.

Bueckner, H., 1970. Novel principle for the computation of stress intensity factors. Zeitschrift fuer Angewandte Mathematik \& Mechanik 50.

Cagnetti, F., Dal Maso, G., Scardia, L., Zeppieri, C.I., 2019. Stochastic homogenisation of free-discontinuity problems. Archive for Rational Mechanics and Analysis 233, 935-974.

Chandler, M., Meredith, P., Brantut, N., Crawford, B., 2016. Fracture toughness anisotropy in shale. Journal of Geophysical Research: Solid Earth 121, 1706-1729.

Chateau, C., Nguyen, T.T., Bornert, M., Yvonnet, J., 2018. DVC-based image subtraction to detect microcracking in lightweight concrete. Strain 54, e12276.

Chopin, J., Bhaskar, A., Jog, A., Ponson, L., 2018. Depinning dynamics of crack fronts. Physical Review Letters 121, 235501.

Chopin, J., Prevost, A., Boudaoud, A., Adda-Bedia, M., 2011. Crack front dynamics across a single heterogeneity. Physical Review Letters 107, 144301.

Clayton, J.D., Knap, J., 2014. A geometrically nonlinear phase field theory of brittle fracture. International Journal of Fracture 189, 139-148.

Dal Maso, G., Toader, R., 2002. A model for the quasi-static growth of brittle fractures:existence and approximation results. Archive for Rational Mechanics and Analysis 162, 101-135.

Delarue, A., Jeulin, D., 2011. Multi-scale simulations of spherical aggregates. Image Analysis \& Stereology 20, 181186. 
Démery, V., Lecomte, V., Rosso, A., 2014a. Effect of disorder geometry on the critical force in disordered elastic systems. Journal of Statistical Mechanics: Theory and Experiment 2014, P03009.

Démery, V., Rosso, A., Ponson, L., 2014b. From microstructural features to effective toughness in disordered brittle solids. EPL (Europhysics Letters) 105, 34003.

Deudé V., Dormieux L., Kondo D., Maghous S., 2002. Micromechanical approach to nonlinear poroelasticity: Application to cracked rocks. Journal of Engineering Mechanics 128, 848-855.

Dimas, L., Bratzel, G., Eylon, I., Buehler, M., 2013. Tough composites inspired by mineralized natural materials Computation, 3d printing, and testing. Advanced Functional Materials 23, 4629-4638.

Dormieux, L., Kondo, D., Ulm, F.J., 2006. Microporomechanics. John Wiley \& Sons.

Evans, A.G., Faber, K.T., 1981. Toughening of ceramics by circumferential microcracking. Journal of the American Ceramic Society 64, 394-398.

Faber, K.T., Evans, A.G., 1983. Crack deflection processes - i. theory. Acta Metallurgica 31, 565-576.

Gao, H., Rice, J., 1986. Shear stress intensity factors for a planar crack with slightly curved front. Journal of Applied Mechanics 53, 774-778.

Gao, H., Rice, J., 1989. A first-order perturbation analysis of crack trapping by arrays of obstacles. Journal of Applied Mechanics 56, 828-836.

Grabois, T., Neggers, J., Ponson, L., Hild, F., Toledo Filho, R., 2018. On the validation of integrated DIC with tapered double cantilever beam tests. Engineering Fracture Mechanics 191, 311-323.

Griffith, A., 1921. The phenomena of rupture and flow in solids. Phil. Trans. R. Soc. Lond. A 221.

Gurtin, M., Podio-Guidugli, P., 1998. Configurational forces and a constitutive theory for crack propagation that allows for kinking and curving. Journal of the Mechanics and Physics of Solids 46, 1343-1378.

Hashin, Z., Shtrikman, S., 1963. A variational approach to the theory of the elastic behaviour of multiphase materials. Journal of the Mechanics and Physics of Solids 11, 127-140.

He, M., Hutchinson, J., 1989. Crack deflection at an interface between dissimilar elastic materials. International Journal of Solids and Structures 25, 1053-1067.

Herve, E., Zaoui, A., 1993. n-layered inclusion-based micromechanical modelling. International Journal of Engineering Science 31, 1-10.

Hill, R., 1963. Elastic properties of reinforced solids: Some theoretical principles. Journal of the Mechanics and Physics of Solids 11, 357-372.

Hossain, M.Z., Hsueh, C.J., Bourdin, B., Bhattacharya, K., 2014. Effective toughness of heterogeneous media. Journal of the Mechanics and Physics of Solids 71, 15-32.

Ibarra, A., Roman, B., Melo, F., 2016. The tearing path in a thin anisotropic sheet from two pulling points: Wulff"s view. Soft Matter 12, 5979-5985.

Irwin, G.R., 1962. Crack-extension force for a part-through crack in a plate. Journal of Applied Mechanics 29, 651-654

Kolton, A.B., Bustingorry, S., Ferrero, E.E., Rosso, A., 2013. Uniqueness of the thermodynamic limit for driven disordered elastic interfaces. Journal of Statistical Mechanics: Theory and Experiment 2013, P12004.

Kolvin, I., Cohen, G., Fineberg, J., 2015. Crack front dynamics: the interplay of singular geometry and crack instabilities Physical Review Letters 114, 175501.

Lachambre, J., Réthoré, J., Weck, A., Buffière, J., 2015. Extraction of stress intensity factors for 3d small fatigue cracks using digital volume correlation and x-ray tomography. International Journal of Fatigue 71, 3-10.

Larkin, A., Ovchinnikov, Y., 1979. Pinning in type II superconductors. Journal of Low Temperature Physics 34, 409-428.

Lebihain, M., 2019. Large-scale crack propagation in heterogeneous materials : an insight into the homogenization of brittle fracture properties. Ph.D. thesis. Sorbonne Université.

Lebihain, M., Leblond, J.B., Ponson, L., 2020. Effective toughness of periodic heterogeneous materials: the effect of out-of-plane excursions of cracks. Journal of the Mechanics and Physics of Solids 137, 103876.

Leblond, J., 1999. Crack paths in three-dimensional elastic solids. i: two-term expansion of the stress intensity factors : application to crack path stability in hydraulic fracturing. International Journal of Solids and Structures 36, 79-103.

Leguillon, D., Tariolle, S., Martin, E., Chartier, T., Besson, J.L., 2006. Prediction of crack deflection in porous/dense ceramic laminates. Journal of the European Ceramic Society 26, 343-349.

Li, Y., Zhou, M., 2013. Prediction of fracture toughness of ceramic composites as function of microstructure: I. numerical simulations. Journal of the Mechanics and Physics of Solids 61, 472-488.

Mandelbrot, B., Passoja, D., Paullay, A., 1984. Fractal character of fracture surfaces of metals. Nature 308, 721-722.

Milton, G.W., 2002. The theory of composites. Cambridge University Press ,. URL: http://dl.merc.ac.ir/handle/Hannan/3193.

Mirkhalaf, M., Dastjerdi, A.K., Barthelat, F., 2014. Overcoming the brittleness of glass through bio-inspiration and micro-architecture. Nature Communications 5, 3166.

Movchan, A., Gao, H., Willis, J., 1998. On perturbations of plane cracks. International Journal of Solids and Structures 35, 3419-3453.

Nasseri, M.H.B., Mohanty, B., 2008. Fracture toughness anisotropy in granitic rocks. International Journal of Rock 
Mechanics and Mining Sciences 45, 167-193.

Nguyen, T., 2015. Modeling of complex microcracking in cement based materials by combining numerical simulations based on a phase-field method and experimental 3D imaging. Ph.D. thesis. ENPC. URL: https://tel.archives-ouvertes.fr/tel-00541139.

Nguyen, T., Réthoré, J., Yvonnet, J., Baietto, M., 2017a. Multi-phase-field modeling of anisotropic crack propagation for polycrystalline materials. Computational Mechanics 60, 289-314.

Nguyen, T.T., Yvonnet, J., Bornert, M., Chateau, C., Bilteryst, F., Steib, E., 2017b. Large-scale simulations of quasibrittle microcracking in realistic highly heterogeneous microstructures obtained from micro CT imaging. Extreme Mechanics Letters 17, 50-55.

Ortiz, M., 1987. A continuum theory of crack shielding in ceramics. Journal of Applied Mechanics 54, 54-58.

Pallares, G., Ponson, L., Grimaldi, A., George, M., Prevot, G., Ciccotti, M., 2009. Crack opening profile in DCDC specimen. International Journal of Fracture 156, 11-20.

Patinet, S., Alzate, L., Barthel, E., Dalmas, D., Vandembroucq, D., Lazarus, V., 2013a. Finite size effects on crack front pinning at heterogeneous planar interfaces: Experimental, finite elements and perturbation approaches. Journal of the Mechanics and Physics of Solids 61, 311-324.

Patinet, S., Vandembroucq, D., Roux, S., 2013b. Quantitative prediction of effective toughness at random heterogeneous interfaces. Physical Review Letters 110, 165507.

Pensée V., Kondo D., Dormieux L., 2002. Micromechanical analysis of anisotropic damage in brittle materials. Journal of Engineering Mechanics 128, 889-897.

Ponson, L., 2016. Statistical aspects in crack growth phenomena: how the fluctuations reveal the failure mechanisms. International Journal of Fracture 201, 11-27.

Ponson, L., Bonamy, D., 2010. Crack propagation in brittle heterogeneous solids: Material disorder and crack dynamics. International Journal of Fracture 162, 21-31.

Ponte-Castañeda, P., Willis, J., 1995. The effect of spatial distribution on the effective behavior of composite materials and cracked media. Journal of the Mechanics and Physics of Solids 43, 1919-1951.

Ramanathan, S., Ertaş, D., Fisher, D., 1997. Quasistatic crack propagation in heterogeneous media. Physical Review Letters 79, 873-876.

Reis, J.M.L., 2006. Fracture and flexural characterization of natural fiber-reinforced polymer concrete. Construction and Building Materials 20, 673-678.

Renard, F., Cordonnier, B., Kobchenko, M., Kandula, N., Weiss, J., Zhu, W., 2017. Microscale characterization of rupture nucleation unravels precursors to faulting in rocks. Earth and Planetary Science Letters 476, 69-78.

Rice, J., 1985. First-order variation in elastic fields due to variation in location of a planar crack front. Journal of Applied Mechanics 52, 571-579.

Roux, S., Hild, F., 2006. Stress intensity factor measurements from digital image correlation: post-processing and integrated approaches. International Journal of Fracture 140, 141-157.

Roux, S., Hild, F., 2008. Self-consistent scheme for toughness homogenization. International Journal of Fracture 154, $159-166$.

Roux, S., Vandembroucq, D., Hild, F., 2003. Effective toughness of heterogeneous brittle materials. European Journal of Mechanics - A/Solids 22, 743-749.

Schneider, M., 2020. An FFT-based method for computing weighted minimal surfaces in microstructures with applications to the computational homogenization of brittle fracture. International Journal for Numerical Methods in Engineering 121, 1367-1387.

Takei, A., Roman, B., Bico, J., Hamm, E., Melo, F., 2013. Forbidden directions for the fracture of thin anisotropic sheets An analogy with the wulff plot. Physical Review Letters 110, 144301.

Torquato, S., 2002. Random Heterogeneous Materials: Microstructure and Macroscopic Properties. Interdisciplinary Applied Mathematics, Springer-Verlag. URL: https ://www . springer. com/gp/book/9780387951676.

Vasoya, M., Lazarus, V., Ponson, L., 2016. Bridging micro to macroscale fracture properties in highly heterogeneous brittle solids: weak pinning versus fingering. Journal of the Mechanics and Physics of Solids 95, 755-773.

Vasudevan, A., Grabois, T., Cordeiro, G., Toledo Filho, R., Ponson, L., 2019. A new fracture test methodology for the accurate characterization of brittle fracture properties. Submitted for publication

Wang, N., Xia, S., 2017. Cohesive fracture of elastically heterogeneous materials: An integrative modeling and experimental study. Journal of the Mechanics and Physics of Solids 98, 87-105.

Widom, B., 1966. Random sequential addition of hard spheres to a volume. The Journal of Chemical Physics 44 3888-3894. 


\section{Appendix A Numerical implementation of the theoretical model}

\section{Microstructure generation.}

Random isotropic non-overlapping microstructures are built using the so-called random sequential addition algorithm proposed by Widom (1966) which consists in placing randomly and sequentially non-overlapping spheres in a fixed volume. This procedure works efficiently for low densities (up to 30\%) or highly polydisperse microstructures. For higher densities and low diameter dispersion levels, we use the algorithm proposed by Delarue and Jeulin (2011) that consists in starting from a dense ordered cubic close-packing of inclusions, and then randomizing it by deleting or moving some of them. These efficient methods permit to generate large-scale isotropic disordered microstructures (typically $10^{6}$ inclusions) within short computation times. Some examples of the generated microstructures are shown in Fig. 8, Fig. 16, and Fig. 18.

Explicit scheme for crack propagation in heterogeneous brittle materials.

The computation of the crack evolution employs an explicit scheme that predicts the configuration of the front at time $t+\Delta t$ from its configuration at time $t$. The crack front is discretized into $N$ points $\left(M_{i}\right)_{i \in[1, N]}$ separated by a uniform distance $\Delta z=\frac{L_{z}}{N}$. First, the angular distribution of the local ERR $G^{i}(\theta)$ is inferred from the instantaneous macroscopic loading $G^{\infty}(t)$ after Eq. (1) and the pertubed SIF $\left(K_{p}^{i}\right)$, computed by a Fast Fourier Transform (FFT) from the current front position $\left(x+f_{x}^{i}, f_{y}^{i}, z_{i}\right)$ using Eq.(3). It is then compared to the angular distribution of the local toughness $G_{\mathrm{c}}^{i}(\theta)$ to infer the local velocity $v^{i}$ in the direction $\theta^{i}$ in the $\left(x M_{i} y\right)$-plane from the kinetic law of Eq. (6) and the GMERR criterion of Eq. (4). The time step $\Delta t$ is finally estimated from a Courant-Friedrichs-Lewy condition combined with an acceleration procedure based on the physics of depinning Lebihain et al. (2020).

\section{Appendix B Estimation of the dissipated energy during crack propagation}

The proposed numerical method allows us to determine the density field $G_{\mathrm{c}}^{\mathrm{frac}}(z, x)$ of dissipated energy from the rate of energy released during propagation. As pictured in Fig. 21, we first discretize the mean fracture plane $(z O x)$ with cells of size $\Delta \ell$. For a grid cell located in $\left(x_{i}, z_{j}\right)$, we note $t_{\text {in }}$ and $t_{\text {out }}$ the time at which the crack front respectively enters and exits the cell (Fig. 21.(a)). Using Griffith's criterion $G=G_{\mathrm{c}}$ valid during crack propagation $(v>0)$, the energy dissipated by fracture during propagation reads:

$$
E_{i, j}^{\mathrm{frac}}=\int_{t_{\mathrm{in}}}^{t_{\mathrm{out}}} \int_{z_{j}-\Delta \ell / 2}^{z_{j}+\Delta \ell / 2} G(z, t) v(z, t) d z d t
$$

where $v(z, t)$ is the instantaneous crack speed along the local crack propagation direction.

We have to normalize this dissipated energy by the cracked surface area. Due to out-of-plane excursions of the crack front, a crack does not propagate over a distance $\Delta \ell$ but instead over a distance:

$$
\Delta s(z)=\int_{t_{\mathrm{in}}}^{t_{\mathrm{out}}} v(z, t) d t
$$




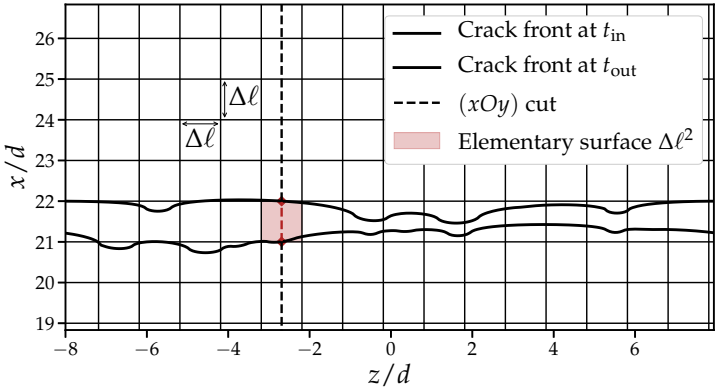

(a) $(z O x)$ plane

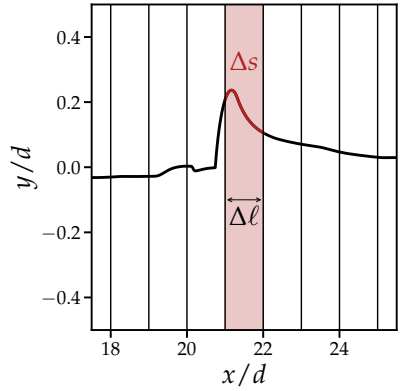

(b) (xOy) plane

Figure 21: Effective fracture energy density, defined as the energy per unit surface required to crack an elementary surface in the $(z O x)$ plane (a) taking into account the out-of-plane excursions (b).

due to the tortuosity of the crack (see Fig. 21). However, we chose to normalize the local dissipated energy $E_{i, j}^{\text {frac }}$ by $\Delta \ell^{2}$ rather than $\Delta \ell \cdot \Delta s$. The field of dissipated energy then reads:

$$
\left[G_{\mathrm{c}}^{\mathrm{frac}}\right]_{i, j}=\frac{1}{\Delta \ell^{2}} E_{i, j}=\frac{1}{\Delta \ell^{2}} \int_{t_{\mathrm{in}}}^{t_{\mathrm{out}}} \int_{z_{j}-\Delta \ell / 2}^{z_{j}+\Delta \ell / 2} G(z, t) v(z, t) d z d t
$$

This procedure is used to to construct local maps of dissipated energy $G_{\mathrm{c}}^{\text {frac }}(z, x)$ such as the one pictured in Fig. 5.

\section{Appendix C Statistics of the fracture energy and the macroscopic loading}

As stated in Section 3.1, the field $G_{\mathrm{c}}^{\text {frac }}$ of dissipated surface energy, which is a local quantity set by the instantaneous ERR $G(z, t, \theta)$, while $G^{\infty}$ is a macroscopic quantity related to the farfield loading. Their instantaneous values are thus expected to be significantly different. This is confirmed in Fig. 22, where are plotted the probability density functions of $G_{{ }_{c}}^{\text {frac }}$ and $G^{\infty}$.

(a)

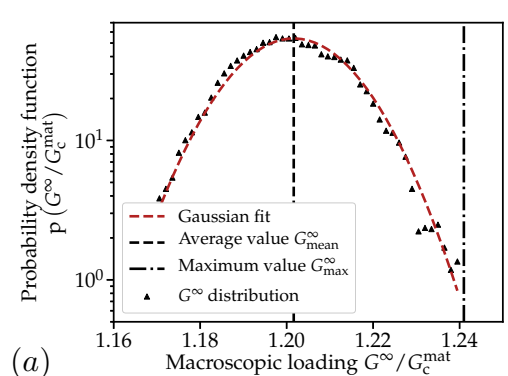

Figure 22: Probability density function of (a) the macroscopic loading and of (b) the effective fracture energy for a crack interacting with a large number of randomly distributed inclusions of density $\rho_{\text {inc }}=25 \%$ and toughness $G_{\mathrm{c}}^{\mathrm{inc}}=2 G_{\mathrm{c}}^{\mathrm{mat}}$. Note that this markedly different statistics have the same average values $\left\langle G_{\mathrm{c}}^{\mathrm{frac}}\right\rangle=G_{\text {mean }}^{\infty}$.

Crack propagation in disordered materials is highly intermittent (Bonamy, 2009; Barés et al., 2014) and composed of pinning phases separated by phases of sudden propagation, called "avalanches". 
During these avalanches, the crack visits multiple pinning configurations, which might not be strong enough to arrest the crack for a given macroscopic loading $G^{\infty}(x)$. As shown by Roux and Hild (2008), this peculiar dynamics leads to a Gaussian probability of $G^{\infty}(x)$ centered in $G_{\text {mean }}^{\infty}$ a feature that is observed in our numerical simulations (see Fig. 22.(a)).

The probability density function of the field $G_{\mathrm{c}}^{\text {frac }}$ of dissipated energy, which is plotted in Fig. 22.(b), shows a behavior markedly different from that of the macroscopic loading $G^{\infty}$ in Fig. 22.(a). We can observe two peaks, one centered around the matrix toughness $G^{\text {mat }}$ and another one around the inclusion toughness $G_{\mathrm{c}}^{\text {inc }}$. These two peaks are surrounded by two regimes corresponding to the relaxation of the crack front perturbation out of the defects. The fact that their respective averages of the,$\left\langle G_{\mathrm{c}}^{\mathrm{frac}}\right\rangle$ and $G_{\text {mean }}^{\infty}$, are found equal is then far from trivial, especially in our numerical experiments where out-of-plane excursions of the crack are considered.

\section{Appendix D Impact of the system size $L_{z}$ on the effective toughness of disordered mate- rials}

The intrinsic homogenized toughness of Section 3 is estimated here from numerical simulations performed on finite-size systems that may not be representative of the macroscopic response of the structure as a whole. The decoupling of the homogenization problem from the loading conditions as well as the size $L_{x}$ of the system in the propagation direction $(O x)$ have been explored in Section 3.2. We investigate here the impact of the system size $L_{z}$ on the effective toughness.

We consider a semi-infinite crack propagating in a heterogeneous medium consisting in a homogeneous matrix and a monodisperse distribution of spherical inclusions of diameter $d$ and density $\rho_{\text {inc }}=25 \%$. The width of the system in the $(O z)$ direction varies from $L_{z}=16 d$ to $L_{z}=512 d$. The crack propagates along a distance $L_{x}=588 d$ to allow small-scale systems to visit enough pinning configurations. Moreover, we consider three inclusion toughness levels $G_{\mathrm{c}}^{\text {inc }} / G_{\mathrm{c}}^{\text {mat }} \in\{1.5,2,3\}$, while the interface toughness remains equal to the one of the matrix $G_{\mathrm{c}}^{\text {int }}=G_{\mathrm{c}}^{\text {mat }}$. Results are plotted in Fig. 23 .
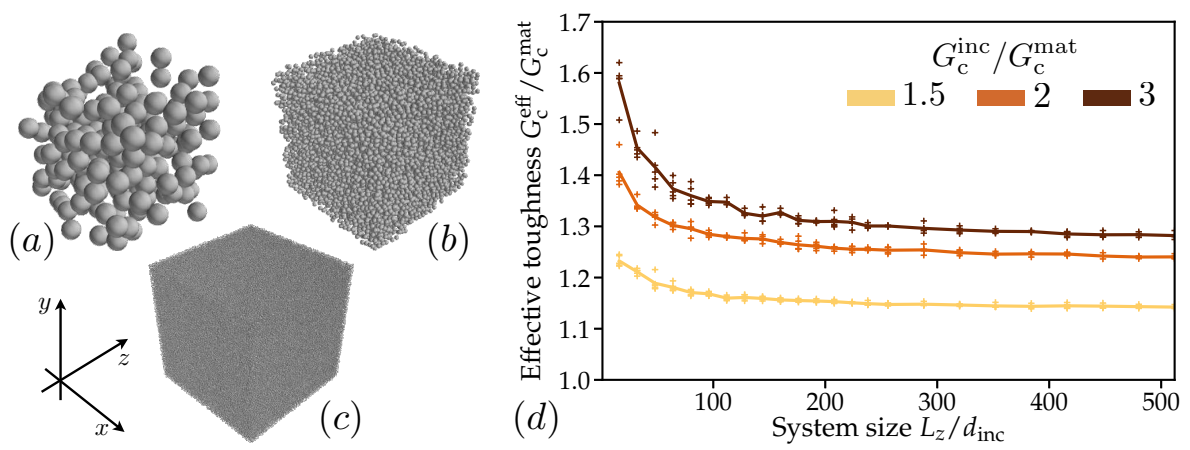

Figure 23: Impact of the system size $L_{z}$ on the effective toughness $G_{\mathrm{c}}^{\mathrm{eff}}$ for the coupling of crack trapping and crack deflection: (a-c) the crack interacts with large-scale distributions of spherical inclusions with varying system size. (d) The effective toughness is estimated from the crack evolution following Eq. (7).

We notice that the effective toughness increases for small $L_{z}$ but converges toward a constant value for large system size, which corresponds to the intrinsic effective toughness of the disordered material. The initial toughening for small system size can be rationalized using the work 
of Démery et al. (2014b): size-dependent effects arise when the system size $L_{z}$ is of the order of the Larkin length $L_{c} \sim\left(\frac{\left\langle G_{c}\right\rangle}{\sigma}\right)^{2} \frac{\xi_{x}^{2}}{\xi_{z}}$, which controls the effective toughness of disordered materials. Eq. (10) predicts that the effective toughness can be renormalized following:

$$
\frac{G_{\mathrm{c}}^{\mathrm{eff}}-\left\langle G_{\mathrm{c}}\right\rangle}{\sigma \sqrt{\frac{\xi_{z}}{L_{c}}}}=\max \left(\sqrt{\frac{L_{c}}{L_{z}}}, 1\right)
$$

To confirm this prediction, we run simulations of coplanar propagation ${ }^{9}$ of a crack interacting with an inclusion distribution of toughness $G_{\mathrm{c}}^{\text {inc }}=1.5 G_{\mathrm{c}}^{\text {mat }}$ and density $\rho_{\text {inc }}=25 \%$. This sets the Larkin length to $L_{c} \simeq 75 d$. We consider system size ranging from $L_{z}=8 d$ up to $L_{z}=4096 d$. Results are plotted in Fig. 24.(a) and renormalization of Eq. (30) is performed in Fig. 24.(b). We observe that the normalized toughness $\left(G_{\mathrm{c}}^{\mathrm{eff}}-\left\langle G_{\mathrm{c}}\right\rangle\right) / \sigma \sqrt{\frac{\xi_{z}}{L_{c}}}$ shows indeed a dependence in $\sqrt{\frac{L_{c}}{L_{z}}}$ when $L_{z} \leq L_{c}$.
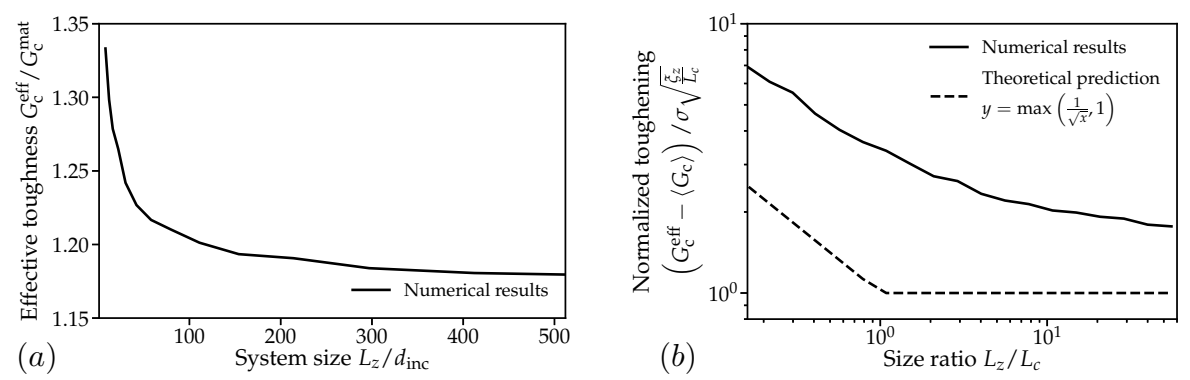

Figure 24: (a) Impact of the system size $L_{z}$ on the effective toughness $G_{\mathrm{c}}^{\text {eff }}$ for coplanar crack propagation. (b) The effective toughness is normalized following Eq. (10).

The presence of a soft transition between the size-dependent $L_{z} \ll L_{c}$ and collective regimes $L_{z} \gg L_{c}$ is nonetheless likely to induce errors in theoretical predictions of the effective toughness for $L_{z} \sim L_{c}$. In the remainder of this paper, we set $L_{z}=256 d$ to ensure that the crack propagates in the collective regime. Note that a large Larkin length implies that the material disorder $\sigma$ is small or that the typical size $\xi_{x}$ of the defects in the propagation direction is large. In that case, $\left\langle G_{\mathrm{c}}\right\rangle$ constitutes a reasonable estimate of the effective toughness (Patinet et al., 2013b).

\section{Appendix $\mathrm{E}$ Impact of the mesh size $\Delta z$ on the effective toughness of disordered materials}

We reproduce the study described in Section 3.3 for various mesh size ranging from $\Delta z=d / 4$ to $\Delta z=d / 32$. Numerical results are plotted in Fig. 25 .

A coarser discretization overestimates the effective toughness, especially at high inclusion toughness levels where by-pass events are predominant. It is due to the fact that a coarser mesh may lead to an overestimation of the deflection angle for inclusion by-pass (see Fig. 25.(b-e)),

${ }^{9}$ Coplanar simulations are computationally more efficient, allowing thus to explore larger system sizes. 
which controls the effective toughness of heterogeneous materials (Brach et al., 2019; Lebihain et al., 2020). As soon as $\Delta z \leq d / 8$ the effective toughness estimates converge towards the same value. We thus adopt a fine mesh $\Delta z \leq d / 16$ for the remainder of this paper, which constitutes a reasonable compromise between computational cost and numerical accuracy.

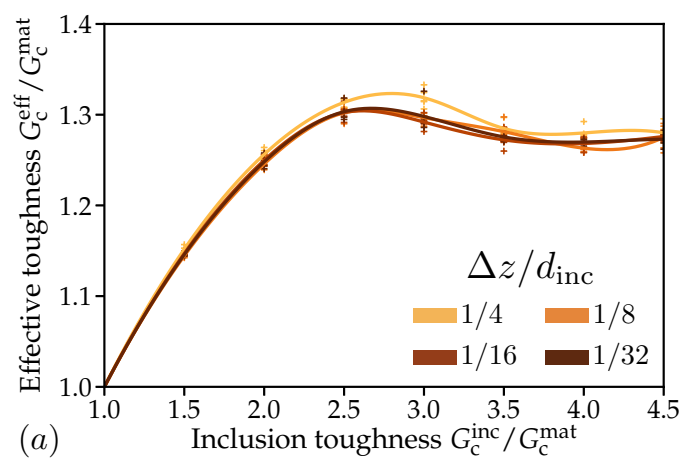

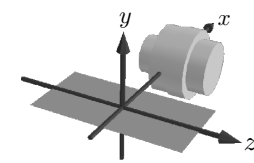

(b)

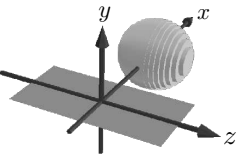

(c)

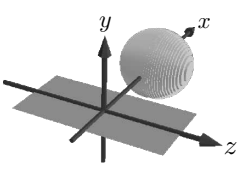

$(d)$

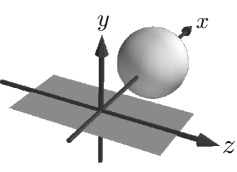

$(e)$

Figure 25: (a) Impact of the front mesh size $\Delta z$ on the effective toughness $G_{\mathrm{c}}^{\text {eff }}$ for the coupling of crack trapping and crack deflection: (b-e) the front is discretized at a varying mesh size $\Delta z$ leading to different inclusion geometries. 